\title{
2D-wavelet Based Micro and Macro Texture Analysis for Asphalt Pavement Under Snow or Ice Condition
}

Feng Li

Beihang University

Gulnigar Ablat

Beihang University

Siqi Zhou (D zsq47@buaa.edu.cn )

Beihang University https://orcid.org/0000-0002-2453-2859

Yixin Liu

Beihang University

Zihang Weng

Tongji University

Yuchuan Du

Tongji University

\section{Research Article}

Keywords: 2D-wavelet, Asphalt pavement, Micro-texture, Macro-texture, Ice, Snow

Posted Date: March 15th, 2021

DOI: https://doi.org/10.21203/rs.3.rs-285832/v1

License: () (1) This work is licensed under a Creative Commons Attribution 4.0 International License.

Read Full License 


\section{D-wavelet based micro and macro texture analysis for asphalt pavement under snow or ice condition}

Feng $\mathrm{Li}^{1}$, Gulnigar $\mathrm{Ablat}^{2}$, Siqi Zhou ${ }^{3 *}$, Yixin $\mathrm{Liu}^{4}$, Zihang Weng ${ }^{5}$, Yuchuan $\mathrm{Du}^{6}$

${ }^{1}$ Professor, School of Transportation Science and Engineering, Beihang University, Beijing 100191, China. Email:

lifeng98@buaa.edu.cn

${ }^{2}$ Master, School of Transportation Science and Engineering, Beihang University, Beijing 100191, China. Email:

gulnigar@buaa.edu.cn

${ }^{3}$ Ph.D. Candidate, School of Transportation Science and Engineering, Beihang University, Beijing 100191, China (corresponding author).Email: zsq47@buaa.edu.cn

${ }^{4}$ Master Candidate, School of Transportation Science and Engineering, Beihang University, Beijing 100191, China. Email:

16131222@buaa.edu.cn

${ }^{5}$ Ph.D. Candidate, Key Laboratory of Road and Traffic Engineering of Ministry of Education, Tongji University, Shanghai 200092, China. Email: 2010184@tongji.edu.cn

${ }^{6}$ Professor, Key Laboratory of Road and Traffic Engineering of Ministry of Education, Tongji University, Shanghai 200092,

China. Email: ycdu@tongji.edu.cn

\section{ABSTRACT}

In ice and snow weather, the surface texture characteristics of asphalt pavement change, which will significantly affect the skid resistance performance of asphalt pavement. In this study, five asphalt mixture types of AC-5, AC-13, AC-16, SMA-13, SMA-16 were prepared under three conditions of the original state, ice and snow. In this paper, a 2D-wavelet transform approach is proposed to characterize the micro and macro texture of pavement. The Normalized Energy (NE) is proposed to describe the pavement texture quantitatively. Compared with the mean texture depth (MTD), NE has the advantages of full coverage, full automation and wide analytical scale. The results show that snow increases the micro-scale texture because of its fluffiness, while the formation of the ice sheets on the surface reduces the micro-scale texture. The filling effect of snow and ice reduces the macro-scale texture of the

\footnotetext{
* Corresponding author
} 
pavement surface. In a follow-up study, the 2D-wavelet transform approach can be applied to improve the intelligent driving braking system, which can provide pavement texture information for the safe braking strategy of driverless vehicles.

Keywords: 2D-wavelet; Asphalt pavement; Micro-texture; Macro-texture; Ice; Snow

\section{INTRODUCTION}

The pavement texture refers to the characteristics of the concave-convex structure on the surface areas, and it is an important index to evaluate the roughness of the pavement surface. The pavement surface texture was subdivided into four ranges based on the wavelength by PIARC (World Road Association 1987): microtexture, macrotexture, megatexture, and unevenness with wavelengths from $0 \mathrm{~mm}$ to $0.5 \mathrm{~mm}, 0.5 \mathrm{~mm}$ to $50 \mathrm{~mm}, 50 \mathrm{~mm}$ to $500 \mathrm{~mm}$ and $500 \mathrm{~mm}$ to $50 \mathrm{~m}$, respectively. It is known from the reports by various studies that the pavement surface texture plays a significant role in the road skid resistance performance. These texture scales have different contributions to tire-pavement friction (Reginald et al. 2016). Typically, a more pronounced depth of macrotexture leads to a better skid resistance performance for roads of the same material (Wang et al. 2013, 2014).

Snow and ice weather have a significant influence on the skid resistance of pavement. In snow and ice weather, the ice or snow attached to the pavement surface will fill the gap of the mixture, significantly reduce the roughness of the pavement surface texture, and significantly change the skid resistance performance of the road surface, which could lead to treacherous driving conditions (Huaxin Chen et al. 2018, Naser et al. 2019, Zafrul et al. 2019).

Pavement surface texture is usually characterized by the mean texture depth (MTD) and the mean profile depth (MPD). Previous research shows that the two parameters are highly correlated (Puzzo et al. 2017). On the one hand, the mean texture depth (MTD) obtained by the sand patch method is not accurate enough to ensure the objectivity of the testing results. On the other hand, the mean profile depth (MPD) by high-speed profiler measurements do not consider all surface profile properties. From what has been discussed above, the two description methods cover many original features of pavement surface texture and fails to meet the needs of current research.

In recent years, various methods have emerged to characteristics of pavement macrotexture accurately (Li Shuo et al._2016, Reginald et al. 2018, Qiang Joshua Li et al. 2018). High-resolution equipments are frequently used to obtain three-dimensional 
texture information of road surface( Wang et al. 2018, Xin et al. 2017, Zhang et al. 2014). Mistakidis used the surface fractal

49

dimension to describe the pavement texture (Mistakidis et al. 1998). De Chen proposed a cost-effective and relatively precise imagebased texture analysis method (ITAM) based on digital image processing and spectral analysis technologies(Chen et al. 2015,2018).

Puzzo Lorenzo used five common cameras to collect pavement surface pictures and calculate the digital Mean Texture Depth (MTD) starting from the Digital Surface Model (DSM) generated by the photos (Puzzo et al. 2017). Liqun Hu used a handy laser scanner to collect 3D texture data of asphalt surface, and eight different roughness parameters were used to describe the 3D characteristics of macrotexture images (Hu et al. 2016). Persson presented a mathematical model that quantitatively determines the kinetic friction coefficient of rubber sliding against a hard, rough substrate, which can be used to predict and calculate skid resistance(Persson 2001). Andreas Ueckermann used a chromatic white light sensor to measure pavement texture and calculated the skid resistance based on the measured texture by means of a rubber friction model(Andreas et al. 2015).

Signal processing methods were applied to analyze the texture data through the researchers' study in recent years (Gendy et al. 2011). Aggregate physical descriptors and Fourier transforms have been used to characterize the texture properties of the aggregates used in asphalt concrete surface courses (Cafiso and Taormina 2007). The power spectral density (PSD) was applied to analyze the pavement texture profile signals (Abbas et al. 2007). However, the advanced data analysis methodologies mask the spatial reference of the data (Kanafi and Tuononen 2017). Wavelet transforms allow frequency domain analysis while preserving spatial reference (Ayenu-Prah and Attoh-Okine 2009). Hence, the wavelet transform was carried out to research the correlation between MPD and wavelet-based parameters (Zelelew et al. 2013, Zelelew et al. 2014, Yang et al. 2018). The wavelet analysis was applied in quantifying aggregate particle surface texture using two-dimensional(2D) grey-scale images (Al-Rousan et al. 2007). Work by Abbas et al. also used wavelet analysis to characterize the 3D surface texture of Portland cement concrete (PCC) cores captured through X-ray computed tomography(Abbas et al. 2017). Yuchuan Du conducted the 2D-wavelet decomposition on eight types of mixtures and used the Relative Energy (RE) and 2D-Entropy to represent the mixture surface texture distribution properties(Du et al. $\underline{2020})$. After making a general survey of studies conducted in the past decades, there is still an obvious limitation that the previous research only focuses on the profile analysis in one direction and neglects the entire surface feature. The pavement surface is a 
surface structure into two-dimensional images and sections. Therefore, the current indicators are the average value of calculating the feature based on a two-dimensional profile, so they lack spatial relations and cannot accurately represent the pavement texture. Because of the above shortcomings, this paper analyzes the three-dimensional pavement surface structure.

This paper aims to implement a two-dimensional discrete wavelet transform to decompose pavement surface texture at micro and macro scales. The 2-D wavelet transform approach separates microtexture and macrotexture into six levels by wavelengths while preserving spatial information. The total energy of each level and the normalized energy (NE) of all surface macro-scale textures are used as the wavelet-based indicators. To evaluate the pavement texture performance under ice or snow condition, we compare the decomposition results of five types of mixtures under three different conditions.

\section{DATA ACQUISITION AND PROCESSING}

\section{Preparation of test specimens}

In this paper, two types of asphalt mixtures (asphalt concrete (AC), stone mastic asphalt (SMA)) were chosen to characterize the pavement texture. Three nominal maximum aggregate sizes (NMAS) were used with mixture AC (AC-5, AC-13, AC-16). At the same time, two NMASs were used with SMA (SMA-13, SMA-16). The forming method of the mixture specimen was the wheel grind method. Figure 1 shows the grading curve of the mixture types.

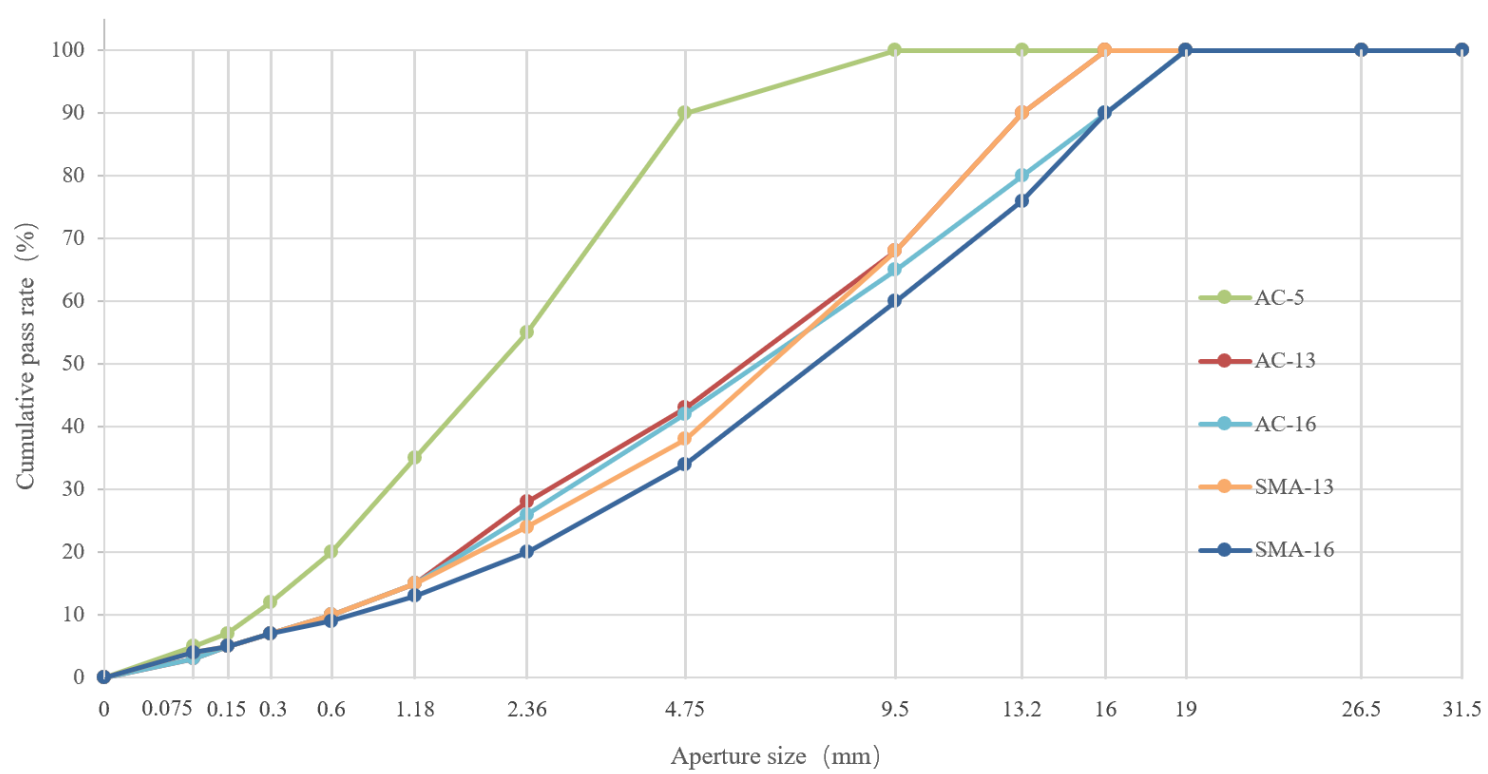


89

simulated test of snow and ice on the five mixtures surface was carried out to describe the influence of snow and ice on the surface texture. When simulating the road surface with ice, water was sprinkled on the surface of the specimens and frozen outdoors at $10{ }^{\circ} \mathrm{C}$ for 1 hour. Considering the rolling action of the wheel on the road surface, the snow was scattered on the surface of the specimens and compacted with a rubber hammer to simulate the texture state of the pavement surface after snow. According to the marked points on the test piece of each specimen, the texture data with the size of $80 \mathrm{~mm} \times 110 \mathrm{~mm}$ was sampled to ensure that the same position was measured every time.

\section{Data acquisition}

In order to measure the surface texture of the specimen, we used a high-resolution three-dimensional profile scanner called VR3000 , which is developed by the company KEYENCE. In this paper, the scanning rate was $12 x$. The scanning accuracy of the apparatus is $1 \mu \mathrm{m}$ for horizontal and $0.5 \mu \mathrm{m}$ for vertical, the sampling frequency of the specimen scanning in this paper is $1 \mathrm{~mm}$. The scanner measured the texture of a $200 \mathrm{~mm} \times 100 \mathrm{~mm}$ area of the surface in one pass. Three-dimensional scanning results of the specimen surface are shown in Figure 2.
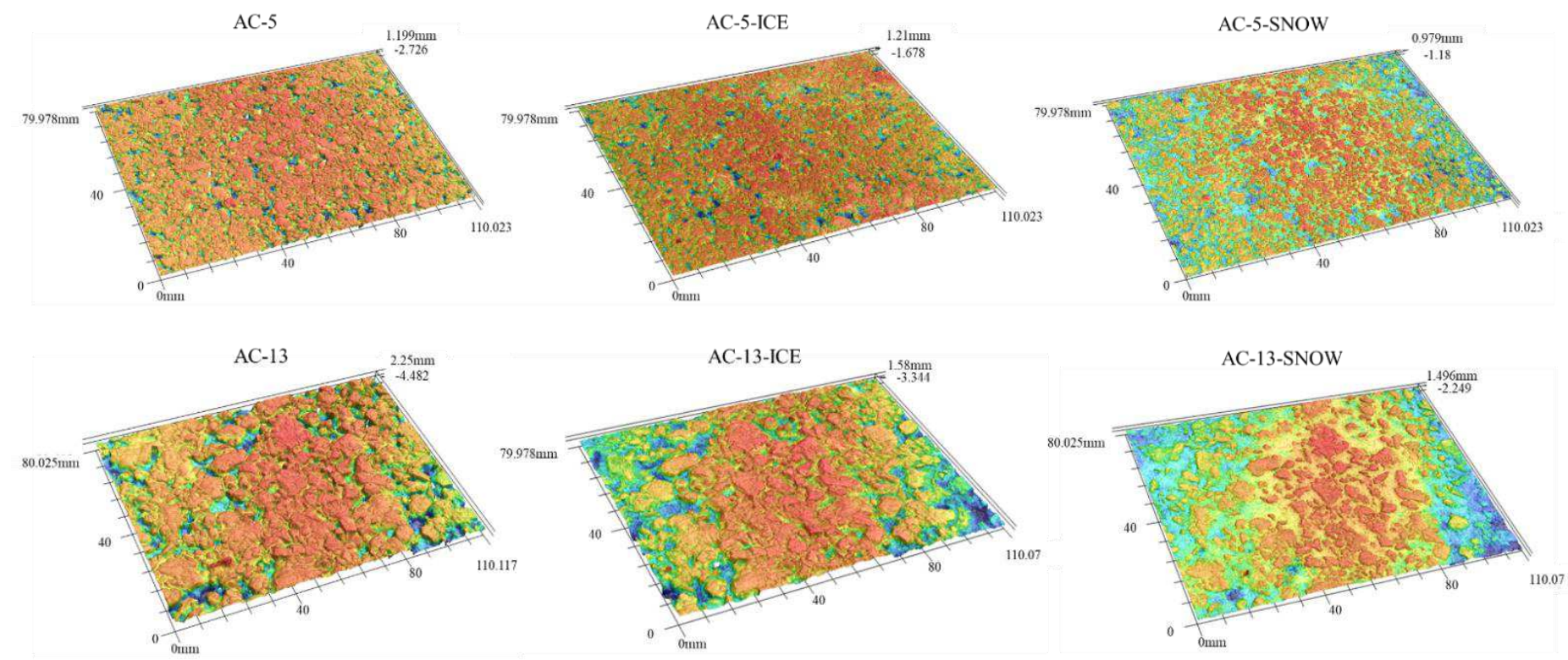

Figure 2 Three-dimensional scanning results of the specimen surface. 

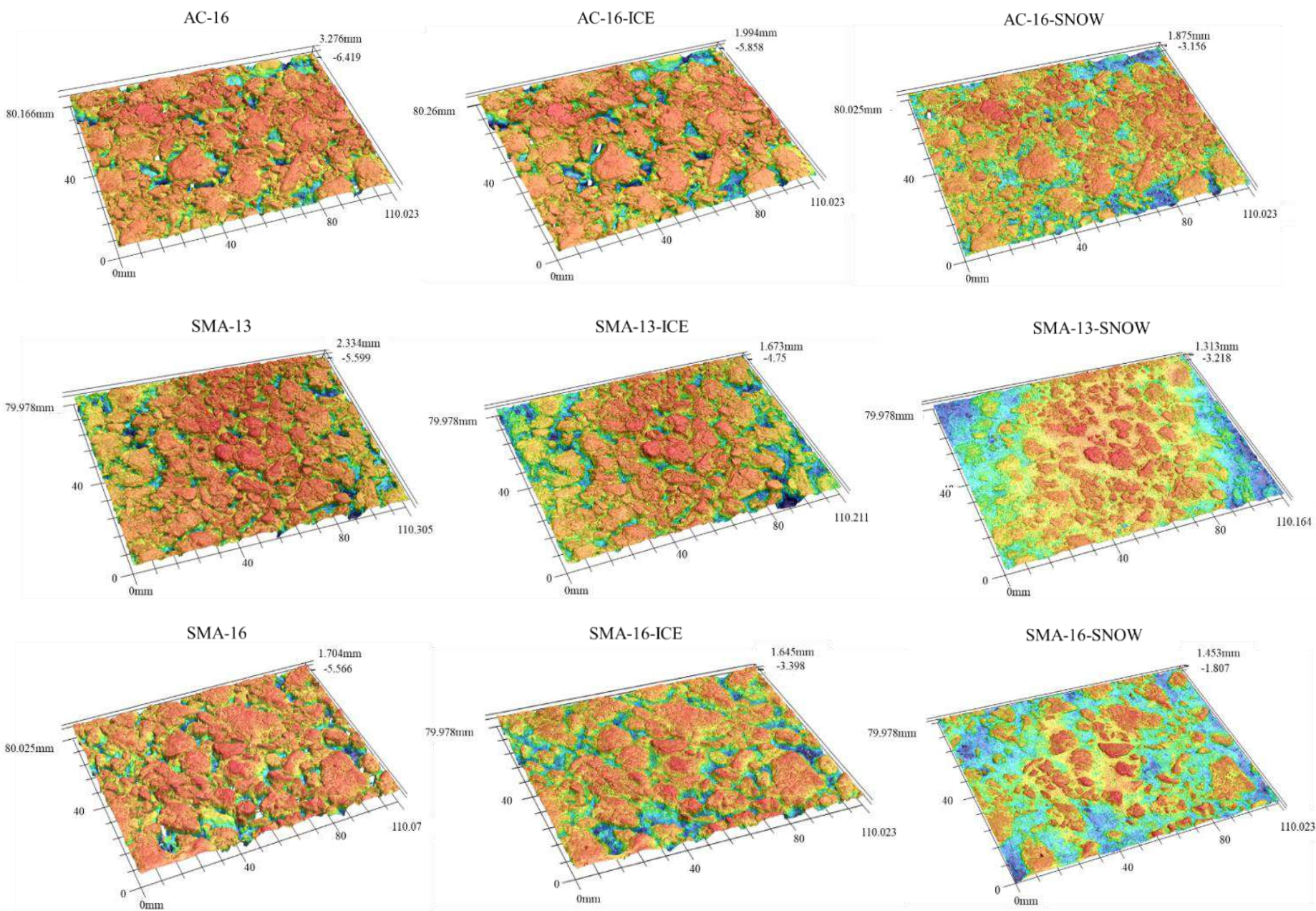

Figure 2 Continued

Because of the roughness of the pavement texture, the scanner will miss some concave data blocked by the convex texture. In

order to ensure the accuracy of the data, we need to use a 2-D filter to remove the outliers in the scanned data. Meshing the point

cloud before the data pre-processing. A $1170 \times 850$ grid is established at $0.1 \mathrm{~mm}$ intervals both in $\mathrm{x}$ - and $\mathrm{y}$-direction. The outliers are

assigned by the interpolation method. Afterward, the data are standardized by subtracting the mean value. A $3 * 3$ sliding window

was applied to deal with the local abnormal. The sliding window moves in the $\mathrm{x}$ and $\mathrm{y}$ plane with a step size of 1 . When the $\mathrm{z}$

variance ( $\mathrm{z}$ means the height of the point) of the points in the sliding window is greater than a certain threshold ( 0.1 in this paper),

the point is regarded to be abnormal, and its value is replaced by the median value of the surrounding points.

The skid resistance performance of the asphalt mixture is closely related to its surface convex and concave distribution, which is

a macroscopic reflection of its microstructure(Huang et al. 2008). The convex points are the main factors of hysteresis on dry roads, and the concave points are the main factors of drain away running water on wet roads. So the concave and convex points are 
considered, but the influence of concave points on drainage is not considered. Kanafi and Tuononen suggest that the analysis from

a top cut of the surface topography on $50 \%$ or less surface area and found a high correlation with friction and top $20 \%$ of PSDs

(Kanafi and Tuononen 2017). In this paper, based on existing practical experience, the top 50\% of the fractal surface was selected as the cutting plane for research and analysis and the top $100 \%$ of the fractal surface was used to make comparative observations, and the $\mathrm{z}$ values below the plane were denoted as zero. Figure 3 shows the fractal surface plane on the different cutting planes.
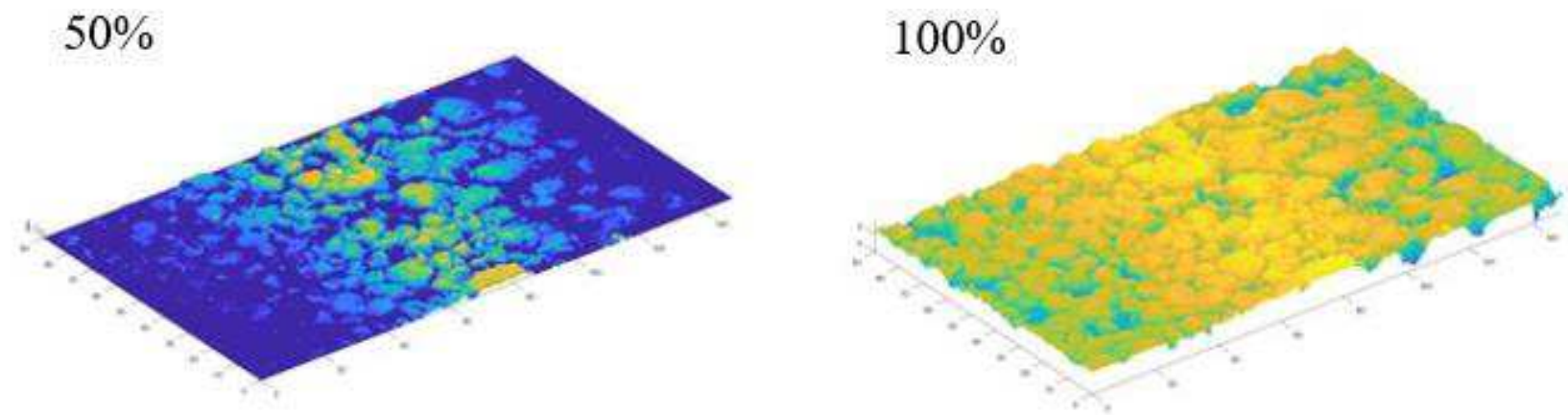

Figure 3 An Example of Fractal Surface on Different Cutting Plane.

\section{Extended measuring method of MTD}

parameters are mean texture depth (MTD), mean profile depth (MPD), and sensor measured texture depth (SMTD). Among them,

MTD is obtained by calculating the ratio of the volume of sand to the average area of the covered circle by the sand patch method.

Although this method is easy to operate and has no special requirements for operators, reproducing the results on one spot is difficult

so that it is not suitable for large-sample testing. At the same time, this method is a fixed-point detection method, which cannot

reflect the macrotexture of the whole region. After the surface profile is generated by the laser detection system, MPD and SMTD can be calculated according to the average algorithm, which can be used to represent the construction depth of the landmark.

However, They have similar limitations to MTD. For example, the use of average algorithms masks some of the macroscopic construction characteristics of the pavement surface.

Based on the uncertainty and unrepeatability of the MTD, a new numerical calculation method is presented. Calculating the volume of a rectangular box between the peak plane and the bottom plane of texture, which is filled with sand and pavement texture, 
according to the following equation:

$$
M T D^{*}=\frac{\left(h_{1}-h_{2}\right) \cdot S-N \cdot V}{S}=\left(h_{1}-h_{2}\right)-\frac{N \cdot V}{S}
$$

where $h 1$ and $h 2$ are, respectively, the height coordinates of the peak and the nadir on pavement texture, $N$ is the number of texture

units, $V$ is the average volume of texture unit, and $S$ is the projected area of surface texture model.

In this study, based on the basic MTD measurement principle that the ratio of the volume of sand and the area of the sand, an

extended method of MTD is proposed. The concave volume can be easily obtained from the software VR-3000, which is the bundled

software with the 3D scanner. As shown in Figure 4, after setting the peak and nadir plane, the volume of the blue area means the

concave diagram. The area of the texture is the scanning area. The MTD is calculated by the following equation:

$$
M T D=\frac{V_{c}}{S}
$$

where $V_{c}$ is the concave volume, and $S$ is the scanning area.

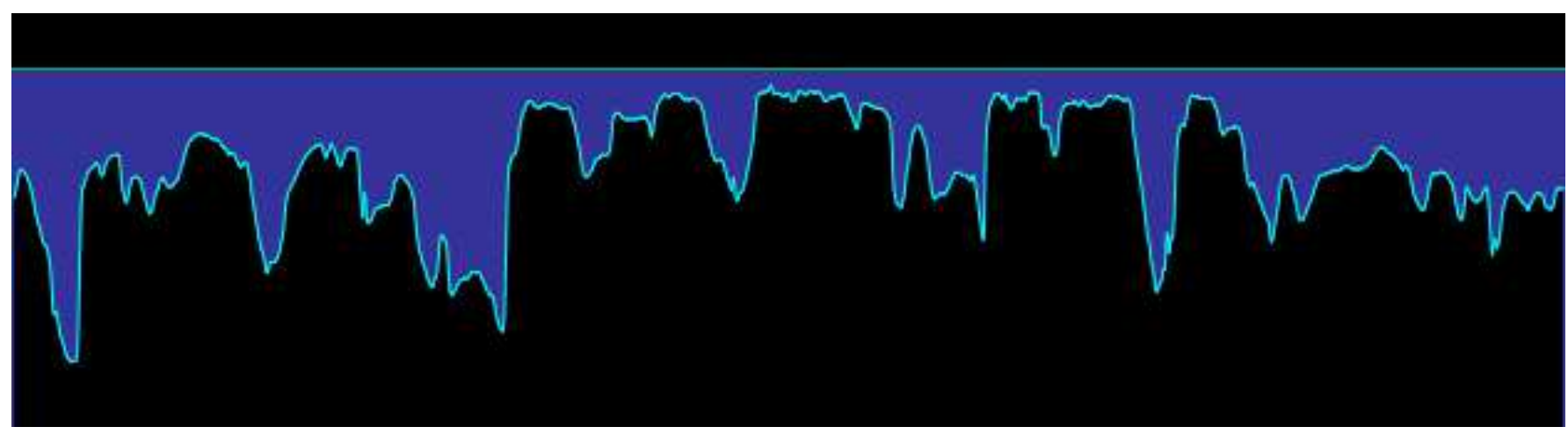

Figure 4 concave volume diagram.

Wavelet Transform

As we all know, the wavelet transform is an improved method of Fourier transform, which is widely used for multi-scale signal

In Mallat's algorithm, the five-level discrete wavelet decomposition can be represented by

$$
\begin{gathered}
\mathrm{s}=a_{1}+d_{1} \\
\mathrm{~s}=\underset{8}{a_{2}}+d_{2}+d_{1}
\end{gathered}
$$




$$
\begin{gathered}
\mathrm{s}=a_{3}+d_{3}+d_{2}+d_{1} \\
\mathrm{~s}=a_{4}+d_{4}+d_{3}+d_{2}+d_{1} \\
\mathrm{~s}=a_{5}+d_{5}+d_{4}+d_{3}+d_{2}+d_{1}
\end{gathered}
$$

The energy is an indicator that measures the overall condition of each part and it can be denoted as:

$$
E=\sum_{x} \sum_{y}\left|z_{x, y}\right|^{2}
$$

160 The energy can also represent the sum of the texture profile elevation with units of length squared(e.g.mm²). The energy (E)

161 characterizes the overall roughness, which varies significantly from different specimens. Moreover, for the same specimen, E is

162 susceptible to slight changes in the detection position. The Total Energy (TE) for all sub-bands (L) can be denoted as:

$$
T E=\sum_{j=1}^{L} E_{j}^{d}
$$

Therefore, the Relative Energy (RE) that indicates the proportion of the energy of each scale, is introduced as:

$$
R E_{i j}=\frac{E_{i j}}{\sum_{i=1}^{n} \sum_{j=1}^{n} E_{i j}},(1 \leq i \leq n, 1 \leq j \leq n)
$$

The Normalized Energy (NE) allows comparisons between total energy statistics obtained from different specimens.

$$
N E=\frac{\sum_{i=1}^{n} \sum_{j=1}^{n} E_{i j}}{N d x d y}
$$

where $d x$ is the distance increment in units of length in the x-direction (e.g., $\mathrm{m}$ ), $d y$ is the distance increment in units of length in

the y-direction (e.g., m).

In this paper, the analysis was carried out using MATLAB Wavelet Toolbox. The selection of the mother wavelet function plays which is composed of 10 compactly supported orthonormal wavelet functions (db1, db2, db3, .., db10). Several studies have used $\mathrm{db} 3$ mother wavelet to analyze pavement surface texture, roughness, and degree of aggregate segregation. The Symlets wavelets are the improved version of $\mathrm{dbN}$. In this paper, sym4 was chosen as the mother wavelet for further analysis. 
176 by decomposing the data in both $\mathrm{x}$ - and y-direction, as shown in Figure 5.

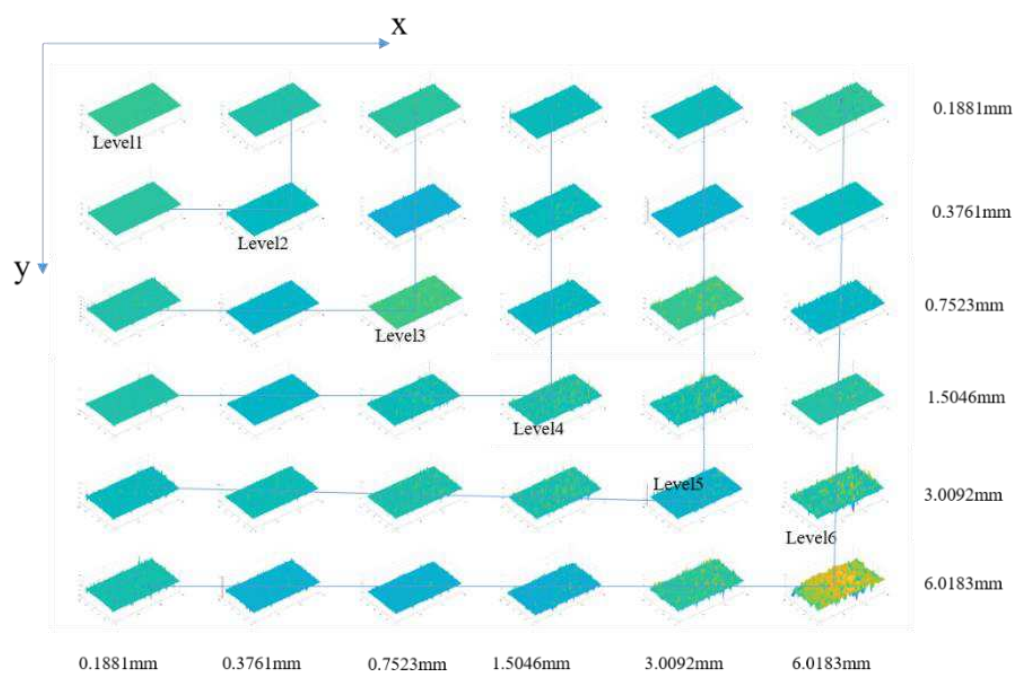

Figure 5 Two-dimensional discrete wavelet transform.

\section{RESULTS AND DISCUSSION}

\section{Determination of MTD}

A comparison between the original specimens and the ice-snow conditions, specimens MTD results is shown in Figure 6. The higher the MTD value, the more complex the macrotexture of the road and the rougher the surface.

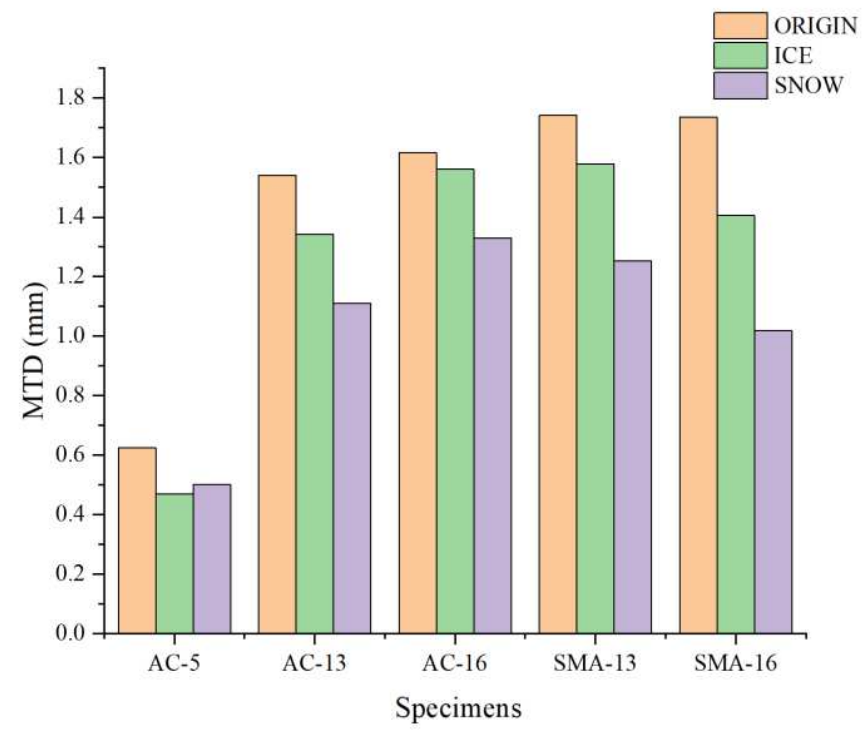

Figure 6 MTD Results.

The texture complexities of different specimens were analyzed by comparing the MTD values of the original surfaces of the five 
specimens in Figure 6. A closer look at the statistic reveals that higher MTD values were measured in SMA specimens, which means

SMA is rougher than AC. Compared with all the specimens, AC-5 had the lowest MTD values and SMA-13 had the highest MTD

values. As can be noticed from Figure 1, the SMA specimens used a gap grinding, which considered a higher percentage of coarse aggregates resulting in higher MTD results. AC-5 had the lowest MTD values because of its minimum nominal particle size.

Normally, SMA-16 should have the highest MTD values; however, because the feature extraction area of the scanner was small, the

texture features of SMA-16 specimens with larger aggregate size were not fully displayed in a small area, so the experimental results showed that SMA-13 had the highest MTD values.

The detailed statistical analysis was asked to identify the changes of MTD values when specimens were in ice or snow conditions.

It can be noticed from Figure 6 that, for most different types of asphalt mixtures, the original surface had the highest MTD value when the surface with snow had the lowest MTD value. The reason why AC-5 had different results from others is when sprayed water on the surface, AC-5 formed an ice sheet on the surface because of its dense structure. At the same time, water sprayed on other specimens permeated into the texture slot and frozen. In general, the MTD value range from $0.469 \mathrm{~mm}$ for the frozen AC-5 specimen surface to $1.741 \mathrm{~mm}$ for the original SMA-13 surface.

\section{Determination of wavelet energy}

The 2D energy matrix was formed as 6*6. The levels in two dimensions are redefined in Figure 5: Level 6 includes the sixth row and column; Level 5 contains the fifth row and column except for the part in Level 6; Level 4 includes the fourth row and column except for the part in Level 5 and 6, and so on. As mentioned above, the 3-D model was respectively cut at the top 50\% and 100\% of a fractal surface plane. Figure 7 Figure 11 show the energy results on the $50 \%$ and $100 \%$ cutting plane in micro- and macroscale. In each of the figures below, The black line represents the original surface, the red line represents the icy surface, and the blue line represents the snowy surface. 

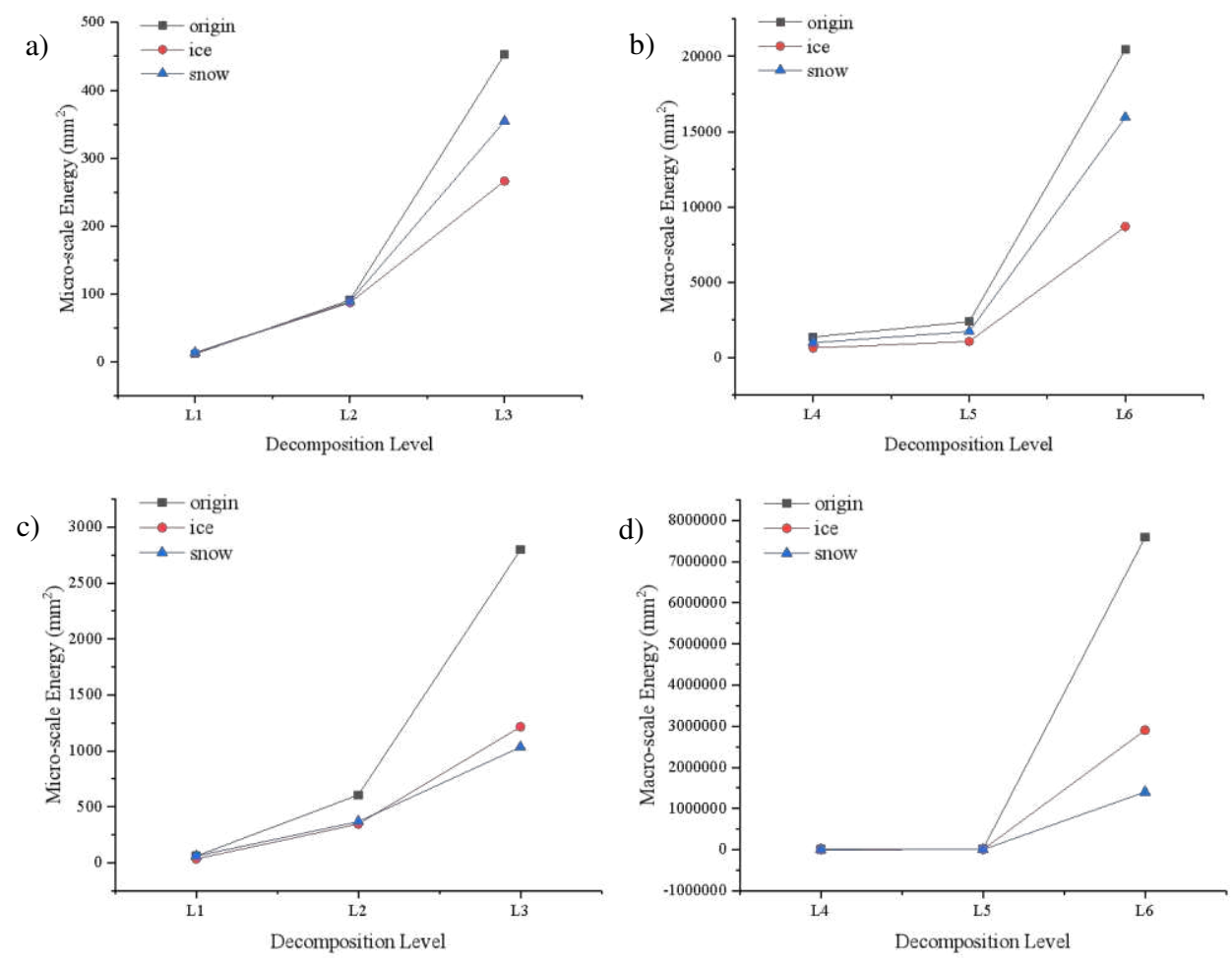

Figure 7 the Energy Results of AC-5; a) 50\% Cutting Plane in micro-scale, b)50\% Cutting Plane in macro-scale, c)100\%

Cutting Plane in micro-scale, d)100\% Cutting Plane in macro-scale.
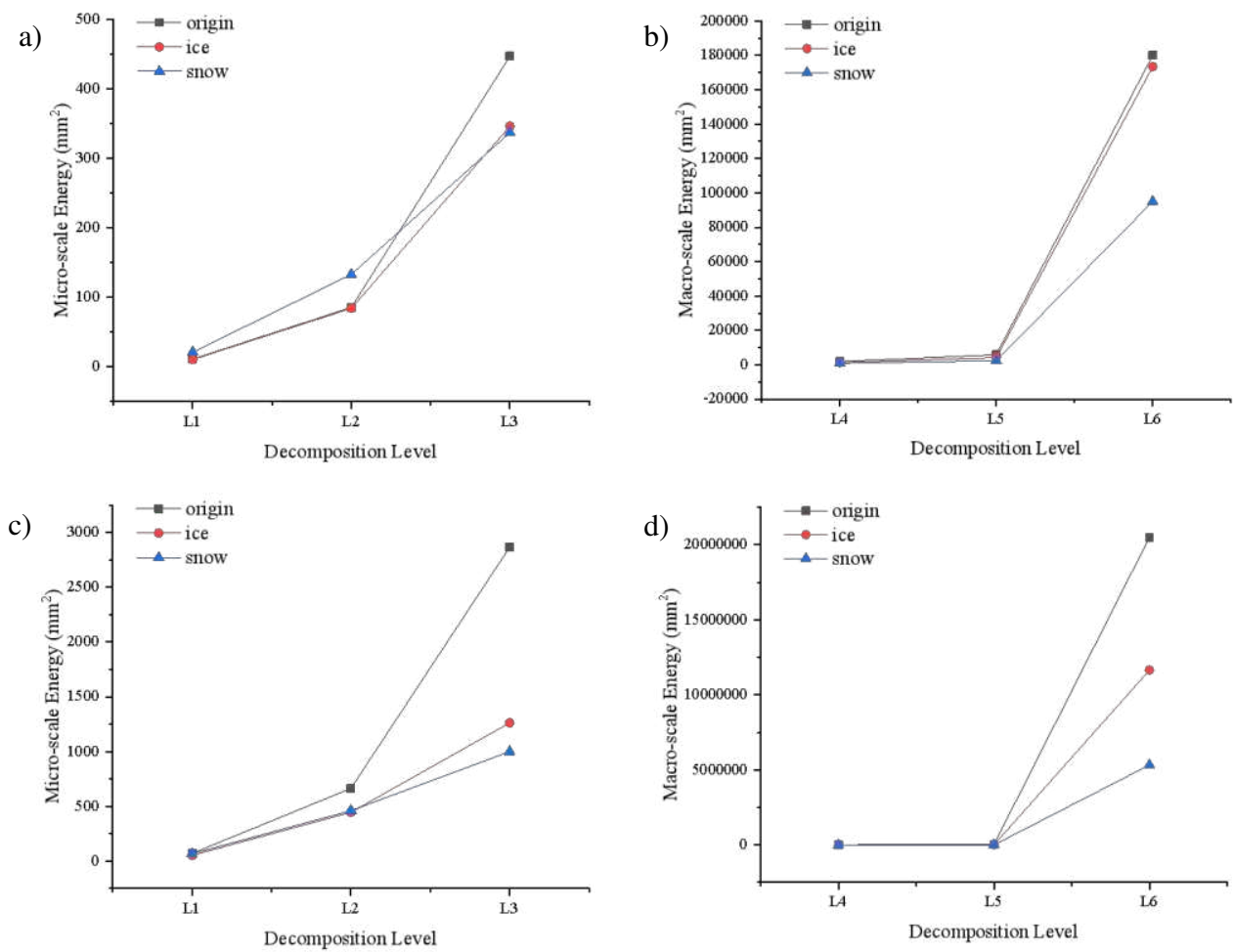

Figure 8 the Energy Results of AC-13; a) 50\% Cutting Plane in micro-scale, b)50\% Cutting Plane in macro-scale, c)100\% 

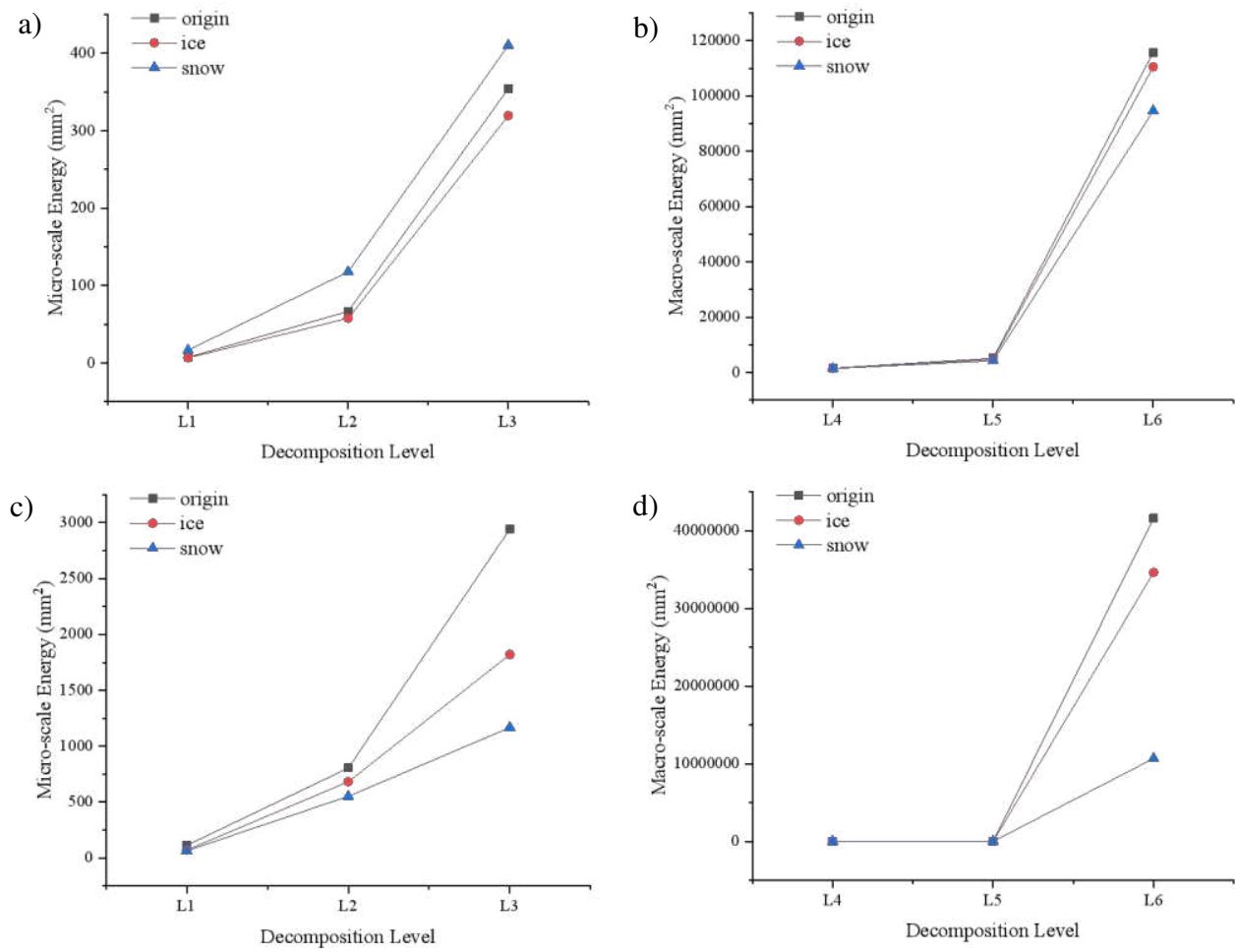

Figure 9 the Energy Results of AC-16; a) 50\% Cutting Plane in micro-scale, b)50\% Cutting Plane in macro-scale, c)100\% Cutting Plane in micro-scale, d)100\% Cutting Plane in macro-scale.
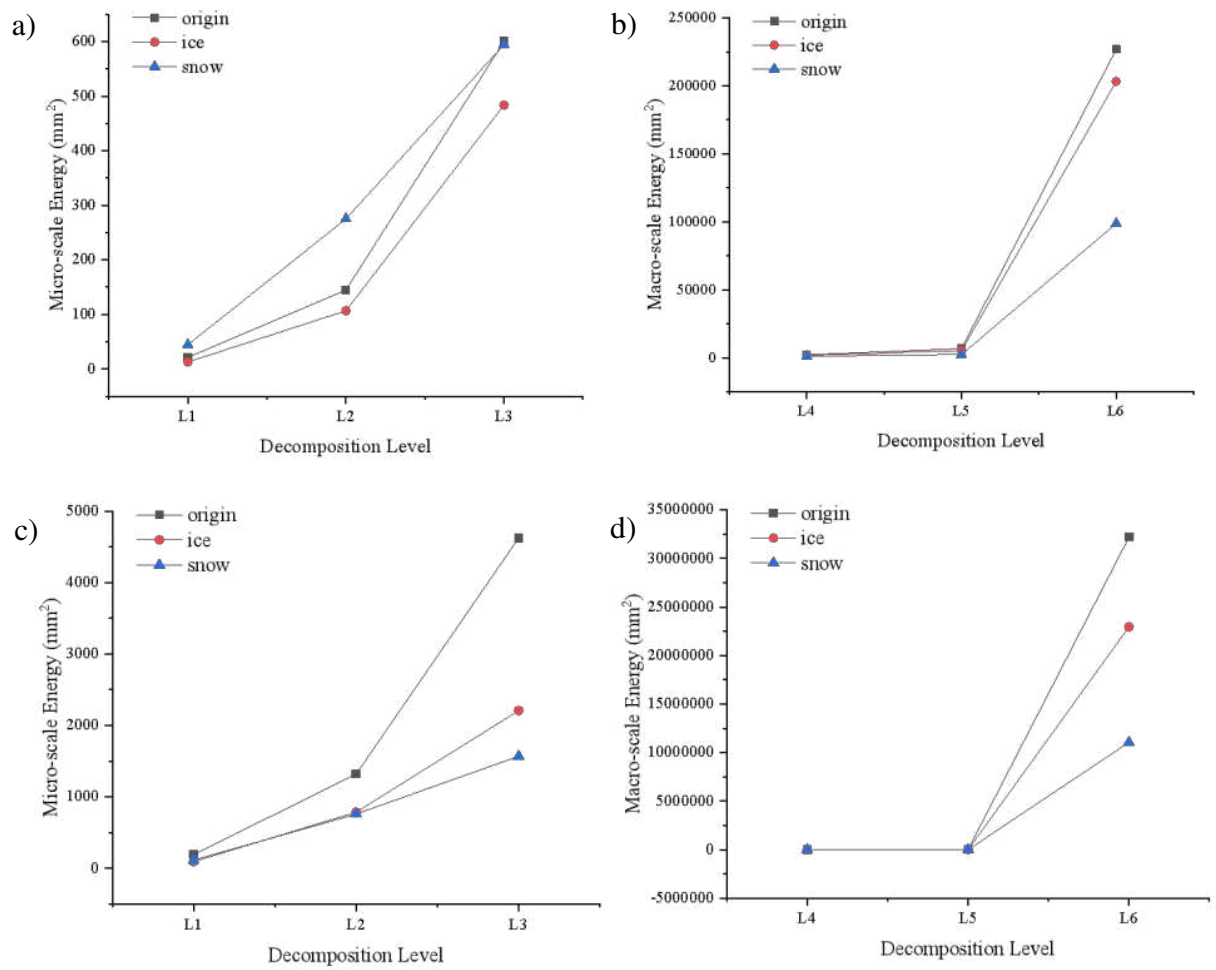

c) $100 \%$ Cutting Plane in micro-scale, d) $100 \%$ Cutting Plane in macro-scale. 
a)
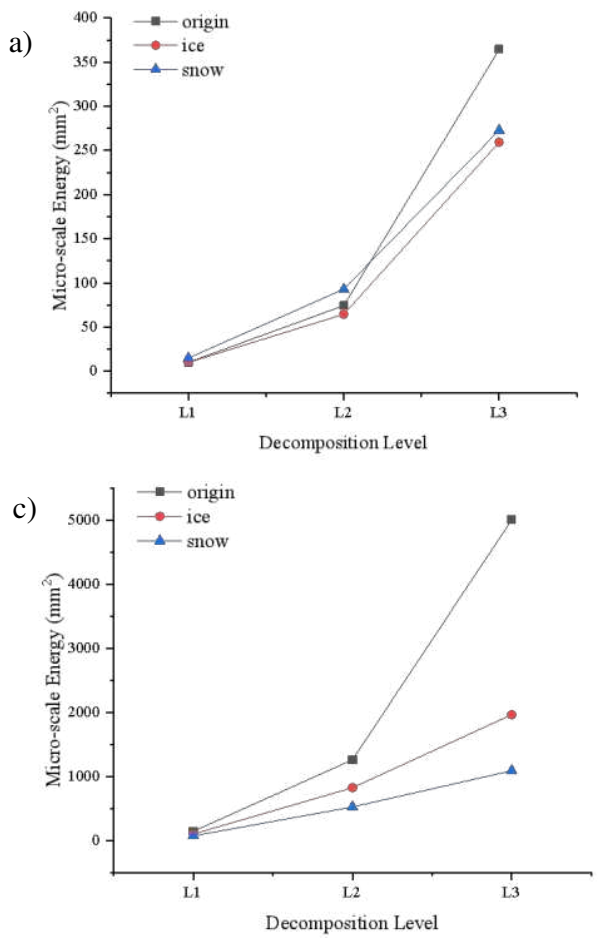

b)

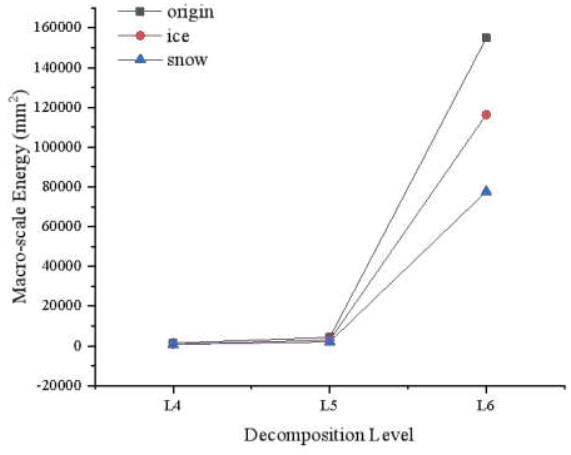

d)

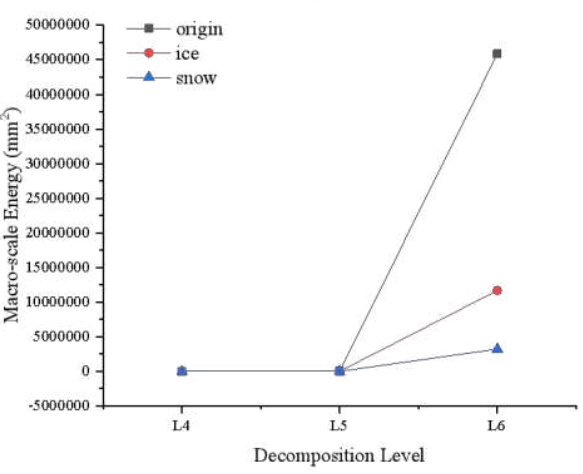

Figure 11 the Energy Results of SMA-16; a) 50\% Cutting Plane in micro-scale, b)50\% Cutting Plane in macro-scale, c) $100 \%$ Cutting Plane in micro-scale, d) $100 \%$ Cutting Plane in macro-scale.

The wavelet energy parameter for each of the decomposition levels can be used to explain the differences in the properties of pavement surfaces. It can be seen from Figure 7 Figure 11 that the micro-scale energy results of AC-5, SMA-13, SMA-16 specimens on the $50 \%$ cutting plane were consistent. On the micro-scale, the original surface showed the highest energy and the icy surface showed the lowest energy. As for the micro-scale energy results of AC-13 specimens, the energy results of icy surface and snow surface were quite similar; the factors that contribute to this situation include the errors generated in the preparation and data collection of specimens. In the micro-scale energy results of AC-16 specimens, the energy value of the snowy surface exceeded that of the original surface. The above characteristics showed that snow increases the micro-scale texture of the pavement surface because of its fluffiness, which leads to severe irregularities on the micro-scale, and the snow surface has abundant micro-scale texture. The icy surface had the lowest energy and the simplest micro-scale texture, which meant the formation of ice sheets on the surface reduced the micro-scale texture.

In Figure 8 Figure 11, it is shown that the macro-scale energy results of AC-13, AC-16, SMA-13 and SMA-16 specimens on the $50 \%$ cutting plane are consistent. The original surface showed the highest energy and the snowy surface showed the lowest 
energy. It indicated that the original surface texture is the most complex, and because of the filling effect of snow and ice on the

234 surface texture, the energy value of the pavement surface was reduced.

In Figure 7 Figure 11, it can be seen that the energy results of each mixture type on the $100 \%$ cutting plane showed the same

change rule no matter on the macro scale or the micro-scale. The original surface showed the highest energy and the snowy surface

237 showed the lowest energy.

\section{Wavelet NE results}

The energy on a single scale is not suitable to represent the overall macrotexture of the pavement surface. Therefore, the

Normalized Energy (NE) was proposed as a new alternative metric to characterize the overall pavement macrotexture. NE is introduced as Equation(7). This statistic represents the sum of the squares of the profile for the wavelengths in the macrotexture range. For the data set analyzed in this paper, it includes wavelengths longer than $0.5 \mathrm{~mm}$ (i.e., wavebands $\mathrm{d}_{4}$ to $\mathrm{d}_{6}$ ).

Figure 12 presents a comparison of wavelet NE values of the selected pavement specimens under various conditions. A Higher

$\mathrm{NE}$ value indicates more complex macrotexture and vice versa. Firstly, the NE values of the original surfaces of various specimens were compared and analyzed. As shown in Figure 12, on the $50 \%$ cutting plane, the maximum value of NE is 26.785 measured by the SMA-13 specimen, and the minimum value of NE is 2.810 measured by the AC-5 specimen. As can be noticed from Figure 1, the SMA specimens used a gap grinding, which considered a higher percentage of coarse aggregates resulting in higher NE results. It is an unexpected result that SMA-16, which has a larger aggregate particle size, has a lower NE value than SMA-13, so as the AC-13 and AC-16. The reason for this result may be due to the limited scanning area of the $3 \mathrm{D}$ profile scanner, that larger particle size reduces the macrotexture of the surface. The NE results on the 50\% cutting plane are consistent with the MTD results of the original surfaces of various specimens mentioned above. On the whole, the NE value of the AC mixture type was less than that of the SMA mixture type. While the results of the $100 \%$ cutting plane of each gradation type show different characteristics from that of the $50 \%$ cutting plane. On the $100 \%$ cutting plane, the maximum value of $\mathrm{NE}$ is 4721.311 measured by the $\mathrm{AC}-16$ specimen and the minimum value of NE is 865.374 measured by the AC-5 specimen. It shows that the texture features of the $50 \%$ cut plane can better represent the macrotexture characteristics of the road surface. 
with the original surface, the NE value of the snowy surface decreases more than that of the ice surface. On the $50 \%$ cutting plane,

and the snow has a more significant impact on pavement texture than ice does.
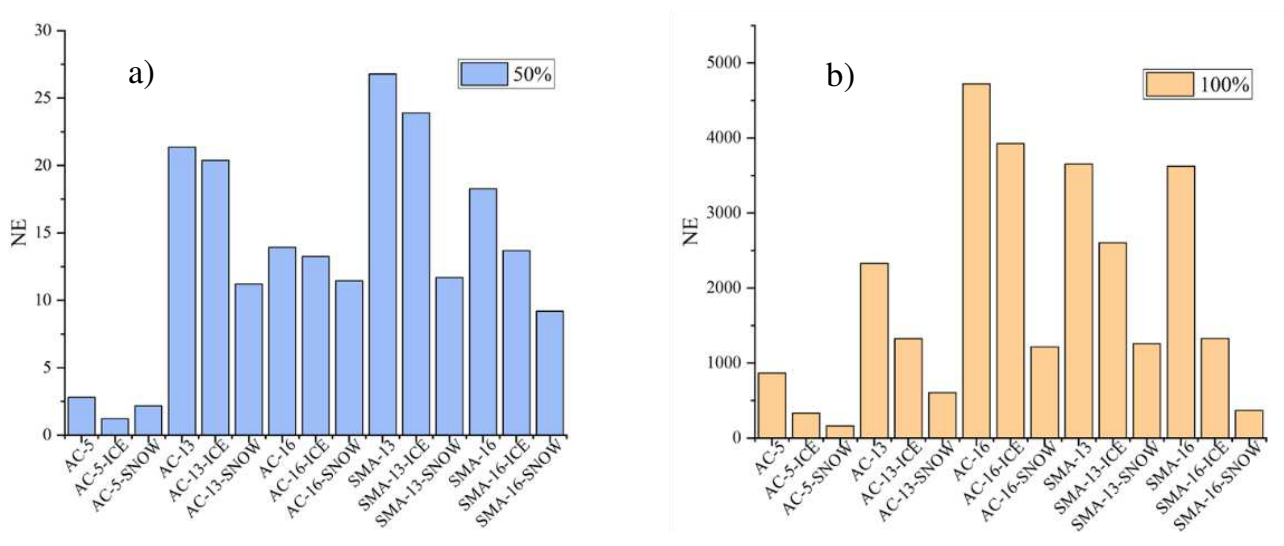

Figure 12 the NE result; a) the NE results on $50 \%$ cutting plane, b) the NE results on $100 \%$ cutting plane.

Comparison between MTD and NE

A simple comparison between MTD and NE values was given in the previous section. The statistical correlation between MTD and NE for each specimen in different conditions was presented in Table 1. The correlation coefficient $\left(\mathrm{R}^{2}\right)$ is used to evaluate the correlation between the two parameters and distinguish the accuracy of their data. The results in this Table suggest that NE is directly related to MTD and can be described by a simple linear relationship (i.e., $y=a x+b)$. It is evident that when a $50 \%$ cutting plane was chosen, the correlation between MTD and NE would show a higher $\mathrm{R}^{2}$ value, which indicates that the texture upper than 50\% height can almost represent the surface macrotexture.

Table 1 Correlation between MTD and NE.

\begin{tabular}{cccc}
\hline Cutting Plane & Condition & Equation & $\mathrm{R}^{2}$ \\
\hline $50 \%$ & Original & $\mathrm{MTD}=0.045 \mathrm{NE}+0.699$ & 0.7616 \\
& Icy & $\mathrm{MTD}=0.045 \mathrm{NE}+0.624$ & 0.7146 \\
& snowy & $\mathrm{MTD}=0.079 \mathrm{NE}+0.324$ & 0.9489 \\
$100 \%$ & Original & $\mathrm{MTD}=0.0003 \mathrm{NE}+0.637$ & 0.7153 \\
& Icy & $\mathrm{MTD}=0.0003 \mathrm{NE}+0.791$ & 0.5806 \\
& snowy & $\mathrm{MTD}=0.0006 \mathrm{NE}+0.633$ & 0.7466 \\
\hline
\end{tabular}

Note: $\mathrm{R}^{2}$ means the correlation coefficient. 
In addition, Figure 13 displays a comparison between MTD and NE on the 50\% cutting plane for each specimen in different

conditions. For simplicity, the MTD values and the NE values were plotted on the same figure. The NE values were shown in the form of a histogram with the vertical axis on the left. The MTD values were shown in the form of a scatter diagram with the vertical axis on the right. For the convenience of observation, the three points under different conditions of the same gradation type were connected into three-point line segments.

It is shown in this figure that the trends (increase, decrease, or no change) in macrotexture properties among the specimens were consistent for both MTD and NE. However, compared with MTD values, the NE values have the advantages of full coverage, full automation and wide analytical scale. Firstly, the variations in the macrotexture properties are better captured using the NE than the MTD. The NE values are calculated based on the entire pavement texture information. In contrast, the MTD values use the sand patch method, a fixed-point detection method, to not reflect the macrotexture of the whole region. Combined with the vehicle driving characteristics in daily life, the wheel paths on the road surface, although concentrated in some locations, also exist and are random in other locations. Therefore, the texture characteristics of the whole pavement surface should be analyzed, which shows that the measurement of pavement texture properties. Thirdly, because of a high-resolution three-dimensional profile scanner and wavelet transform method, NE extracts and analyzes the pavement texture properties on micro and macro scales respectively, and the analysis scale was wider. Above all, it suggests that the wavelet approach is better suited to characterize the macrotexture properties of asphalt pavements. 


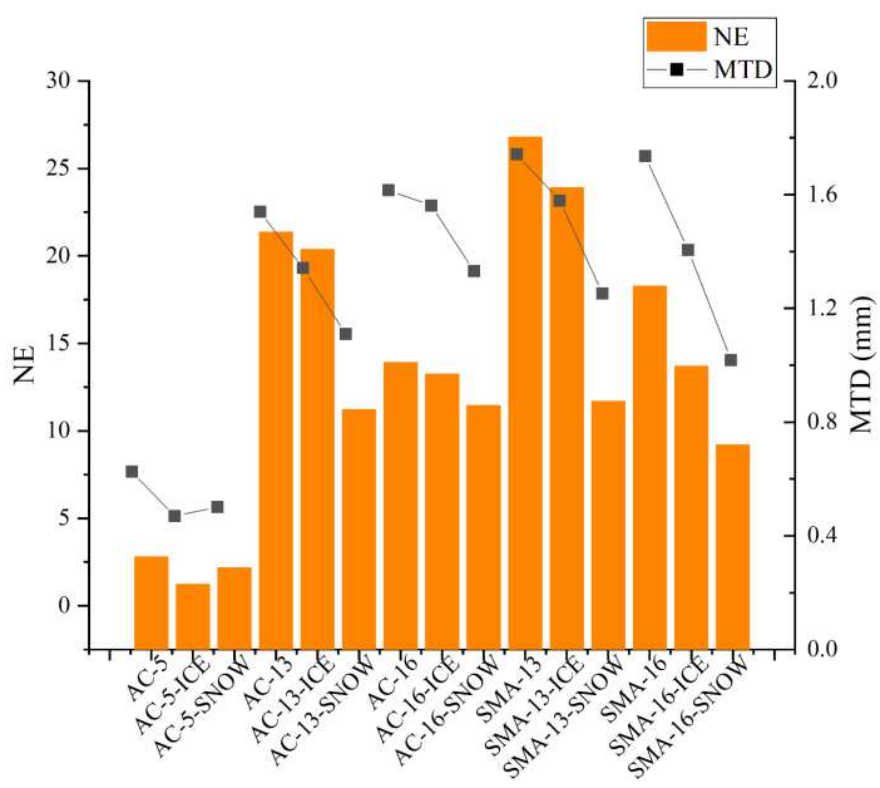

Figure 13 Comparison between MTD and NE for 50\% cutting plane.

\section{CONCLUSIONS}

In this study, a 2D-wavelet transform approach was proposed to decompose the $3 \mathrm{D}$ pavement texture measured by a highresolution 3D profile scanner into micro-scale and macro-scale in two directions. The asphalt pavement specimens consisted of two asphalt mixture types (asphalt concrete (AC) and stone mastic asphalt (SMA)), and the pavement surface was under different conditions (original, icy, and snowy). The surface topography was made a top cut off at $50 \%$ and $100 \%$ height before decomposition. The Normalized Energy (NE) was used as the wavelet-based indicator. Through the observation and data analysis of the experimental results in this paper, the following conclusions were obtained:

(1) The 2D-wavelet transform approach can be used as a tool to make in-depth use of the high-resolution 3D data, and then the road performances, such as skid resistance and degree of wear, can be evaluated. It shows that the Normalized Energy (NE), a macro texture description index based on the 2D-wavelet approach proposed in this paper, has a strong correlation with the traditional index MTD. MTD and NE results show the same trend of macroscopic texture. Compared with MTD values, the NE values have the advantages of full coverage, full automation and wide analytical scale. Therefore, the 2D-wavelet transform approach is more suitable for the characterization of texture properties of pavement surfaces.

(2) The 2D-wavelet transform approach is further applied to analyze the texture properties on the pavement covered with snow and ice. On the micro-scale, original surfaces had the highest micro-scale energy, and icy surfaces had the lowest micro-scale energy. 
The snow increased the micro-scale texture of the pavement surface because of its fluffiness, which led to severe irregularities on the micro-scale, and the snowy surface had abundant micro-scale texture. The formation of the ice sheets on the surface reduced the micro-scale texture, making the texture of the icy surface the simplest. As for the macro scale, the origin surfaces had the highest energy, and the snowy surfaces had the lowest energy. The reason for this was that the filling effect of snow and ice on the surface reduced the macro-scale texture of the pavement surface.

(3) The 2D-wavelet transform approach can be used to extract and analyze the micro and macro texture, and can be applied to both scientific researches and engineering practice in a future study. For example, nowadays, the unmanned drive is a hot topic in today's smart city construction. This approach can be applied to improve the intelligent driving braking system due to its full

traffic loads adequately. Further research can focus on both the macro and micro-texture under different gradation types and working conditions.

\section{DECLARATIONS}

Availability of data and materials

Not applicable. 
manuscript. All authors read and approved the final manuscript.

\section{Acknowledgements}

Not applicable.

\section{REFERENCES}

Abbas, A., Kutay, M..E., Azari, H. Rasmussen, R. (2007) Three-dimensional surface texture characterization of portland cement concrete pavements. Computer Aided Civil \& Infrastructure Engineering, 22(3), 197-209.

Al-Rousan, T., Masad, E., Tutumluer, E., Pan, T. (2007) Evaluation of image analysis techniques for quantifying aggregate shape characteristics. Construction and Building Materials, 21, 978-990.

Andreas, Ueckermann, Dawei, Wang, Markus, \&Oeser, Bernhard Steinauer (2015) Calculation of skid resistance from texture measurements. Journal of Traffic and Transactions Engineering(English Edition), 2(1), 3-16.

Ayenu-Prah, A. Y. and Attoh-Okine, N. O (2009) Comparative study of Hilbert-Huang transform, Fourier transform, and wavelet transform in pavement profile analysis. Vehicle System Dynamics, 47(4), 437-456.

Baotao Huang, Weiping Tian, Jiachun Li, E.Fractal Cui (2008) Method for Quantitative Evaluation of Skid Resistance of Asphalt

Pavement. China Journal of Highway and Transport(Chinese Edition) (04):12-17.

Bruce, Andrew, Donoho, David, Gao, Hong-Ye (1996) Wavelet analysis. IEEE Spectrum, 33(33), 26-35.

Cafiso, S., and Taormina, S. (2007) Texture analysis of aggregates for wearing courses in asphalt pavements. International Journal of Pavement Engineering, 8(1), 45-54.

De, Chen, Nima, R., S., Hussain B. (2015) Exploring the feasibility of evaluating asphalt pavement surface macro-texture using image-based texture analysis method, Road Materials and Pavement Design, 16:2, 405-420.

De, C. (2018) Evaluating asphalt pavement surface texture using 3D digital imaging, International Journal of Pavement Engineering. 
Du, Y., Weng, Z., Li, F., Ablat, G., \& Liu, C. (2020) A novel approach for pavement texture characterisation using 2d-wavelet decomposition. International Journal of Pavement Engineering(6), 1-16.

El, G., A., Shalaby, A., Saleh, M., Flintsch, G (2011) Stereo-vision applications to reconstruct the 3D texture of pavement surface.

International Journal of Pavement Engineering, 12(3), 263-273.

Geometric Product Specifications (GPS) - Surface Texture: Areal, 2012. ISO 25178.

Hall, J.W., Smith, K.L., Titus-Glover, L., Evans, L.D., Wambold, J.C., Yager, T.J., et al. (2009) Guide for Pavement Friction

Contractor's Final Report for National Cooperative Highway Research Program (NCHRP) Project 01-43, 2009.Transportation

Research Board of the National Academies, Washington, D.C.

Hu, L., Yun, D., Liu, Z., Du, S., Zhang, Z., Bao, Y (2016) Effect of three-dimensional macrotexture characteristics on dynamic

frictional coefficient of asphalt pavement surface. Construction and Building Materials, 126, 720-729.

Huaxin Chen, Yongchang Wu, Huiyun Xia, et al (2018) Review of ice-pavement adhesion study and development of hydrophobic surface in pavement deicing. Journal of Traffic and Transportation Engineering (English Edition), 5(3): 224-238.

Kanafi, M. M., and Tuononen, A. J. (2017) Top topography surface roughness power spectrum for pavement friction evaluation.

Tribology International, 107, 240-249.

Kogbara R B, Masad E A, Kassem E, et al. (2016) A state-of-the-art review of parameters influencing measurement and modeling

of skid resistance of asphalt pavements, 2016. Construction and Building Materials, 114(Jul .1):602-617.

Li, S. Harris, D, Wells, T (2016) Surface texture and friction characteristics of diamond-ground concrete and asphalt pavements.

Journal of Traffic \& Transportation Engineering, 3(5), 475-482.

Mistakidis E.S., Panagouli O.K., Panagiotopoulos P.D. (1998) Unilateral contact problems with fractal geometry and fractal friction

laws: methods of calculation. Computational Mechanics, 21(4-5):353-362. 
Persson, B.N.J. (2001) Theory of rubber friction, contact mechanics and the role of surface roughness on the adhesion of elastic

solids. In: 160th Meeting of the Rubber Division, American Chemical Society, Cleveland.

PIARC (1987) Report of the committee on surface characteristics, in Permanent International Association of Road Congress (PIARC)

XVIII World Road Congress, Brussels.

Puzzo, L., Loprencipe, G., Tozzo, C., D'Andrea, A (2017) Three-dimensional survey method of pavement texture using photographic

equipment. Measurement, 111, 146-157.

Qiang, J.L., Zhan Y, Yang G, et al (2018) Pavement skid resistance as a function of pavement surface and aggregate texture properties.

International Journal of Pavement Engineering.

Reginald, B., Kogbara, E., Masad, A., David, W., Phillip, M. (2018) Relating surface texture parameters from close range

photogrammetry to Grip-Tester pavement friction measurements, Construction and Building Materials, Volume 166, Pages 227-240.

Wang, D., Chen, X., Yin, C., Oeser, M., Steinauer, B (2013) Influence of different polishing conditions on the skid resistance development of asphalt surface. Wear, 308(s1-s2), 71-78.

Wang, D., Ueckermann, A., Schacht, A., Oeser, M., Steinauer, B., Persson, B. N. J (2014) Tire-road contact stiffness. Tribology

Letters, 56(2), 397-402.

Wang, Y., Yang, Z., Liu, Y., Sun, L (2018) The characterisation of three-dimensional texture morphology of pavement for describing pavement sliding resistance. Road Materials and Pavement Design, 1-20.

Xin, Q., Qian, Z., Miao, Y., Meng, L., Wang, L (2017) Three-dimensional characterization of asphalt pavement macrotexture using

laser scanner and microelement. Road Materials and Pavement Design, 18(sup3), 190-199.

Yang, G., Li, Q. J., Zhan, Y. J., Wang, K. C. P, Wang, C (2017) Wavelet based macrotexture analysis for pavement friction prediction.

Ksce Journal of Civil Engineering. 
392 Zafrul H. Khan, Md Rashadul Islam, Rafiqul A. Tarefder (2019) Determining asphalt surface temperature using weather parameters.

393 Journal of Traffic and Transportation Engineering (English Edition), 6(6): 577-588.

394 Zelelew, H., Khasawneh, M., and Abbas, A. (2014) Wavelet-based characterization of asphalt pavement surface macro-texture. Road

395 Materials and Pavement Design, 15(3), 622-641.

396 Zelelew, H. M., Papagiannakis, A. T., and Izeppi, E. D. D. (2013) Pavement macro-texture analysis using wavelets. International 397 Journal of Pavement Engineering, 14(8), 725-735.

398 Zhang, X., Liu, T., Liu, C., Chen, Z, (2014) Research on skid resistance of asphalt pavement based on three-dimensional laser399 scanning technology and pressure-sensitive film. Construction and Building Materials, 69, 49-59. 
Figures

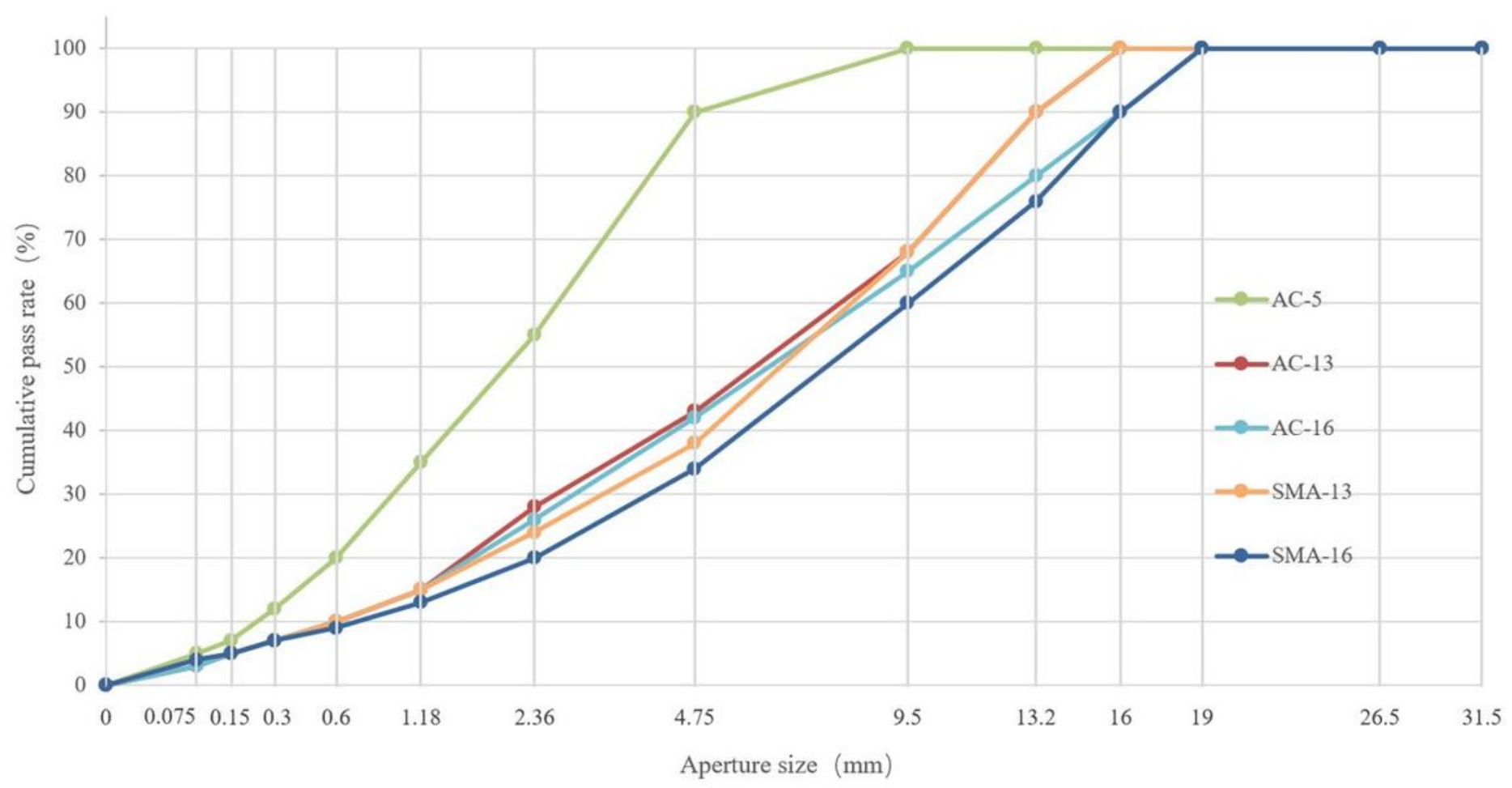

Figure 1

Grading Curve. 

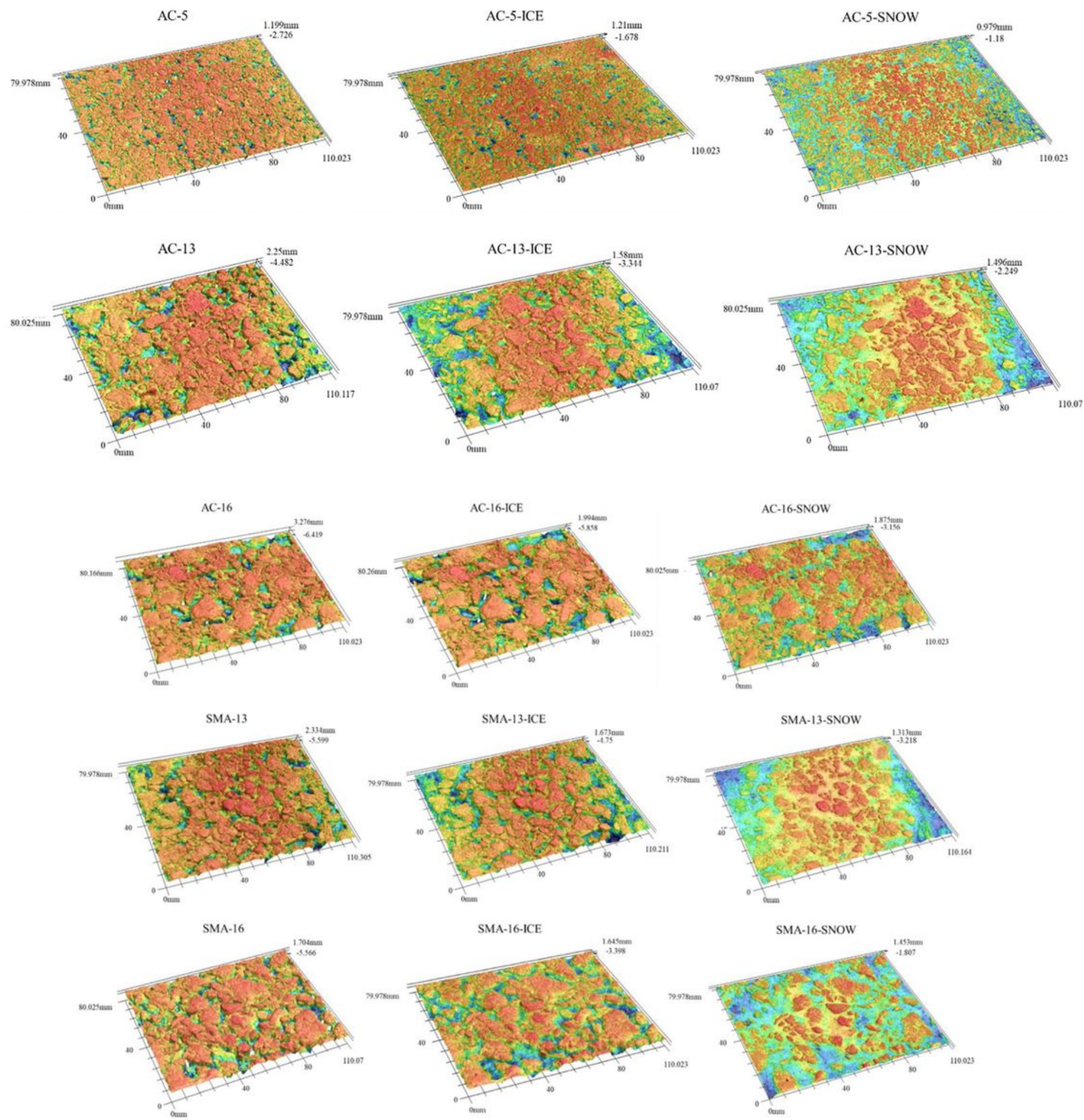

Figure 2

Three-dimensional scanning results of the specimen surface. 

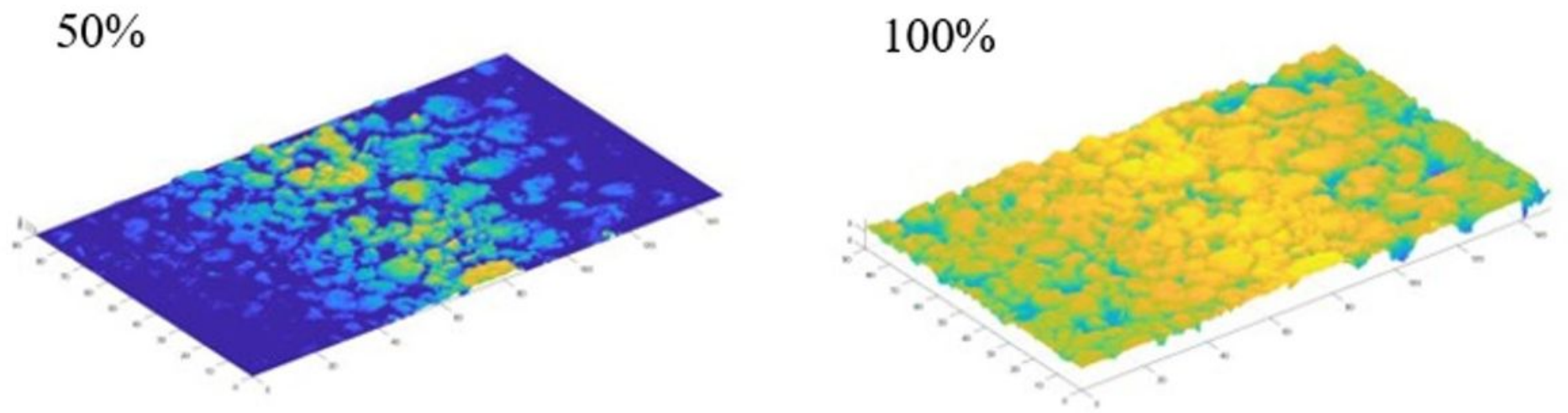

Figure 3

An Example of Fractal Surface on Different Cutting Plane.

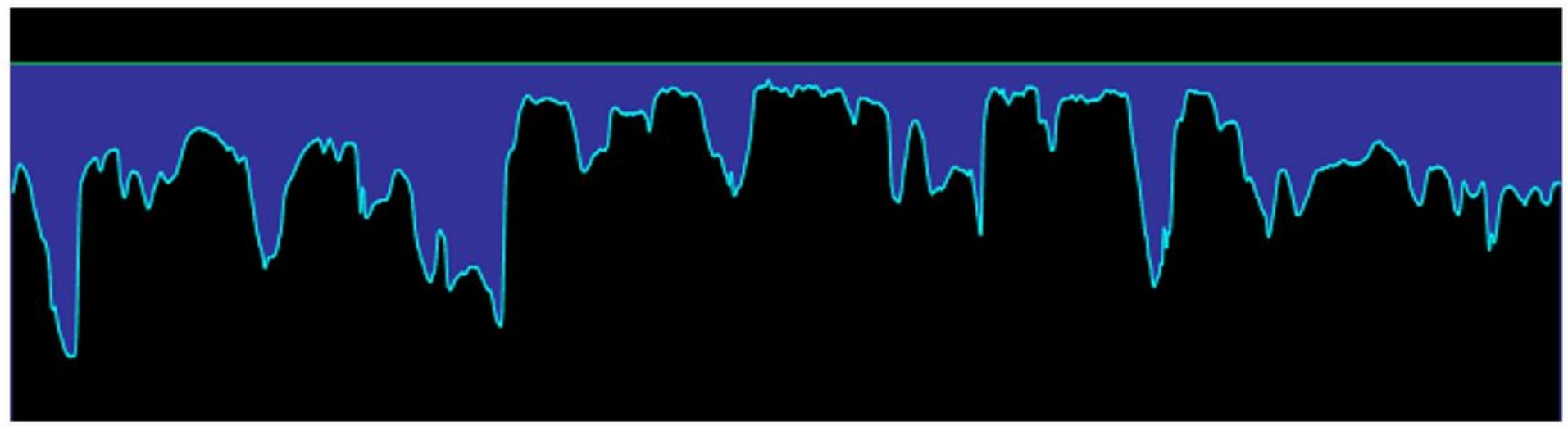

Figure 4

concave volume diagram. 


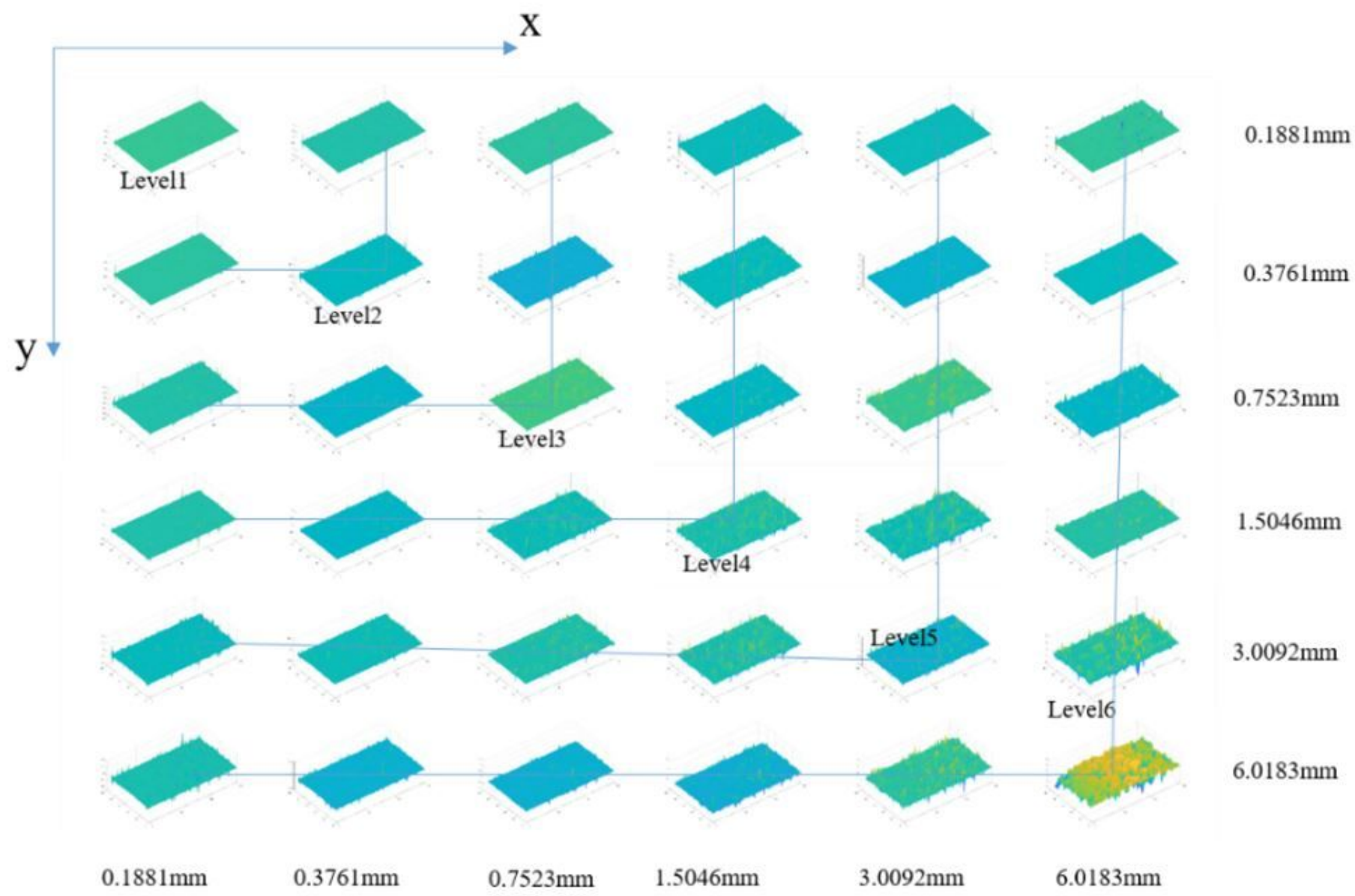

Figure 5

Two-dimensional discrete wavelet transform. 


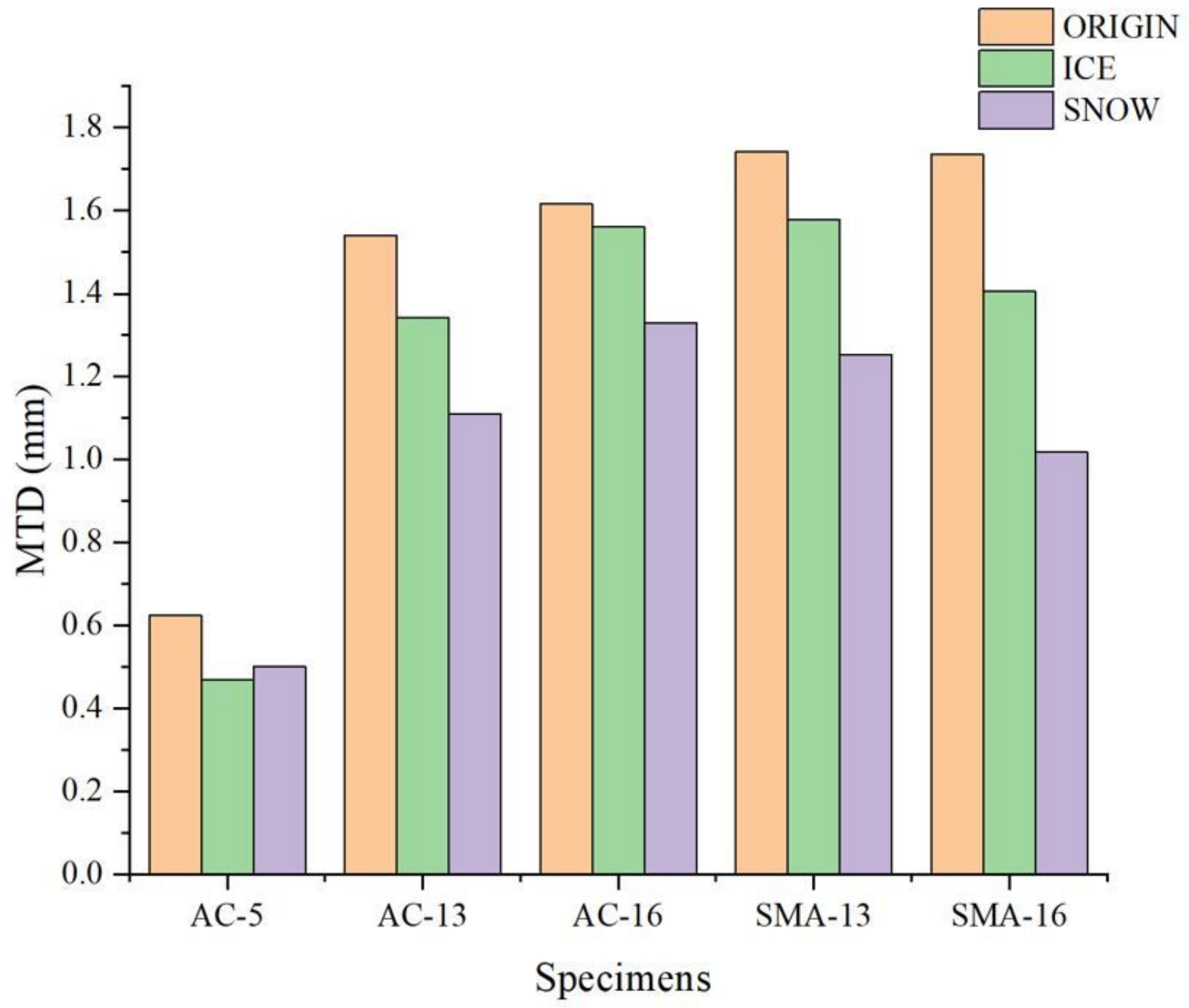

Figure 6

MTD Results. 

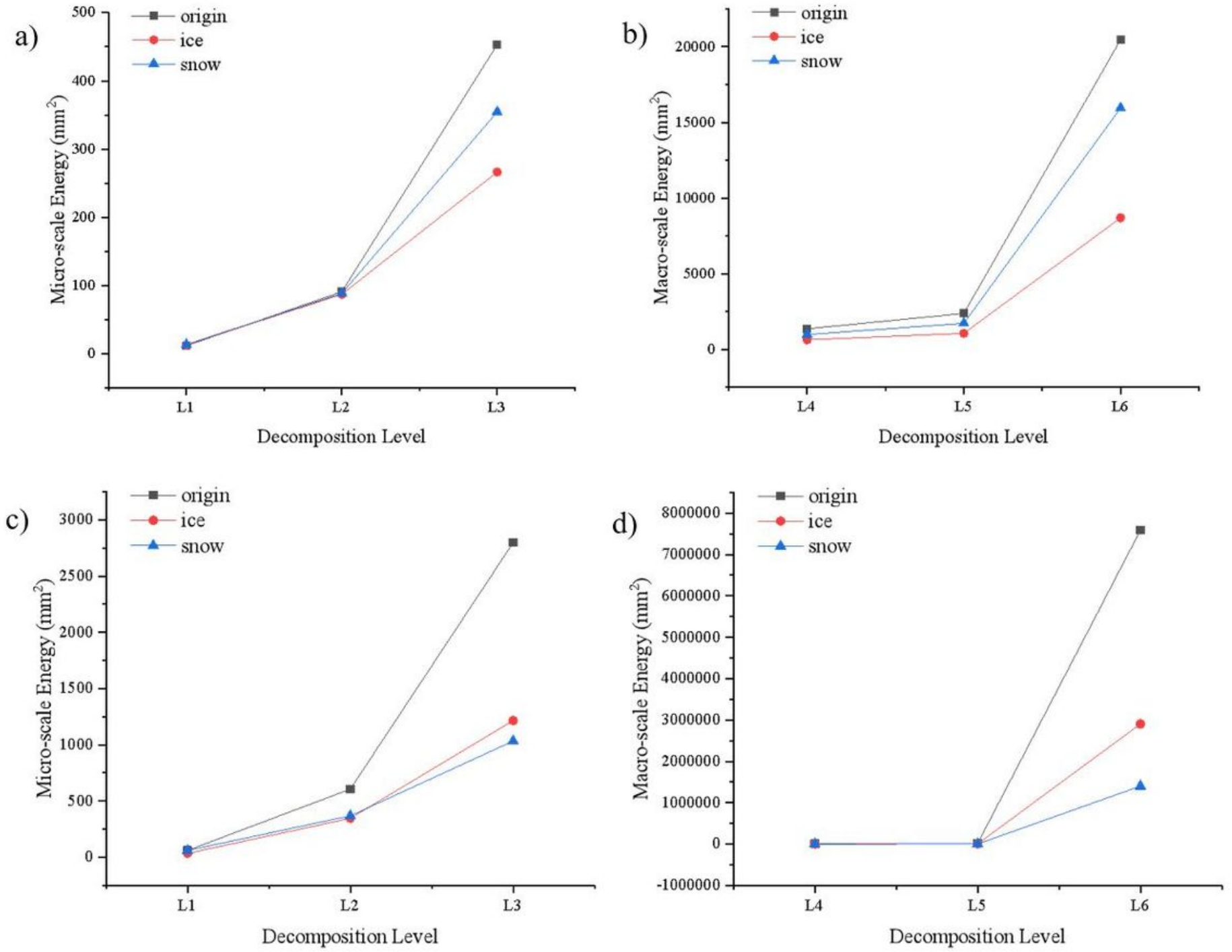

Figure 7

The Energy Results of AC-5; a) 50\% Cutting Plane in micro-scale, b) $50 \%$ Cutting Plane in macro-scale, c) $100 \%$ Cutting Plane in micro-scale, d) $100 \%$ Cutting Plane in macro-scale. 
a)

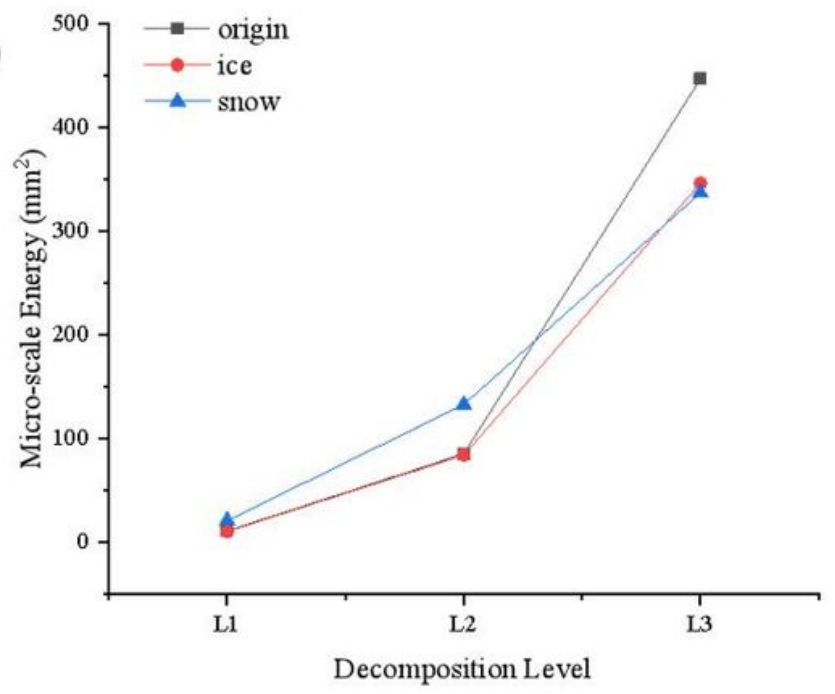

c

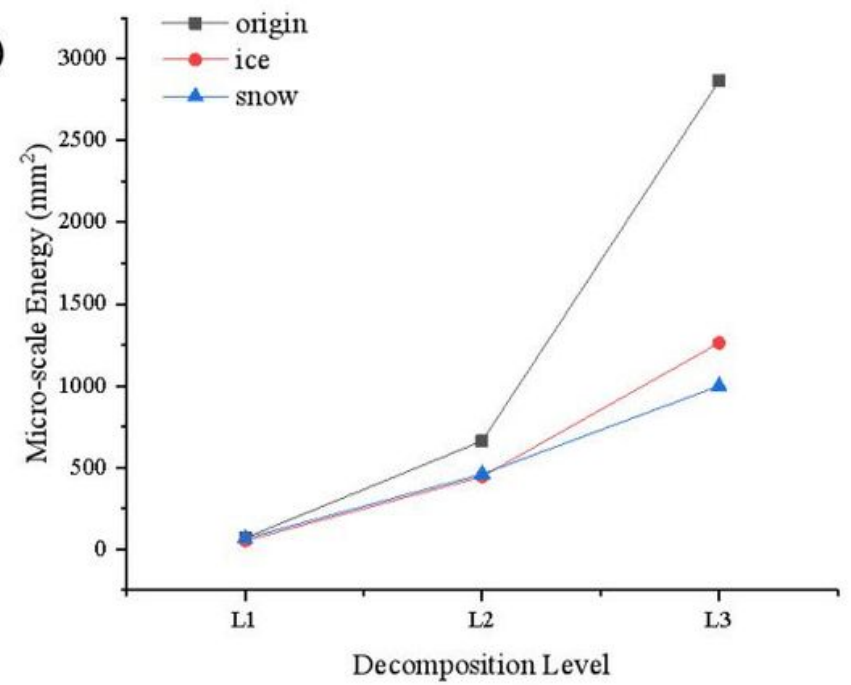

b)

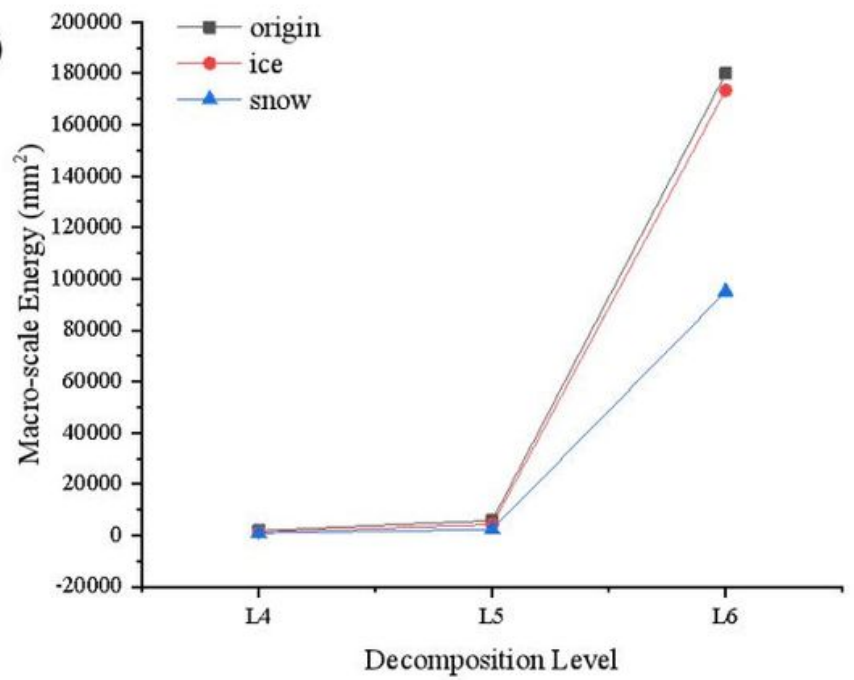

d)

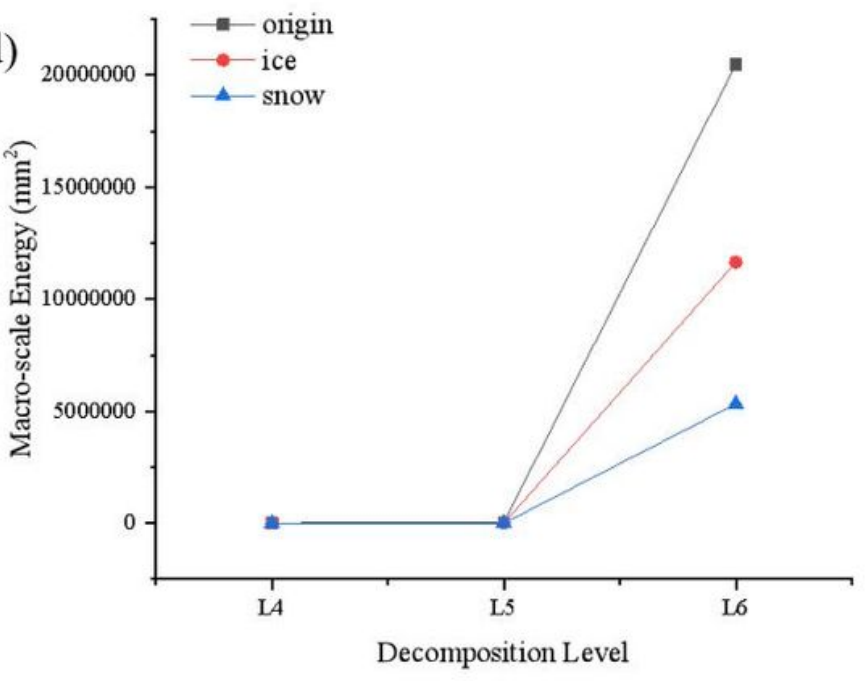

Figure 8

The Energy Results of AC-13; a) 50\% Cutting Plane in micro-scale, b) $50 \%$ Cutting Plane in macro-scale, c) $100 \%$ Cutting Plane in micro-scale, d) $100 \%$ Cutting Plane in macro-scale. 
a)

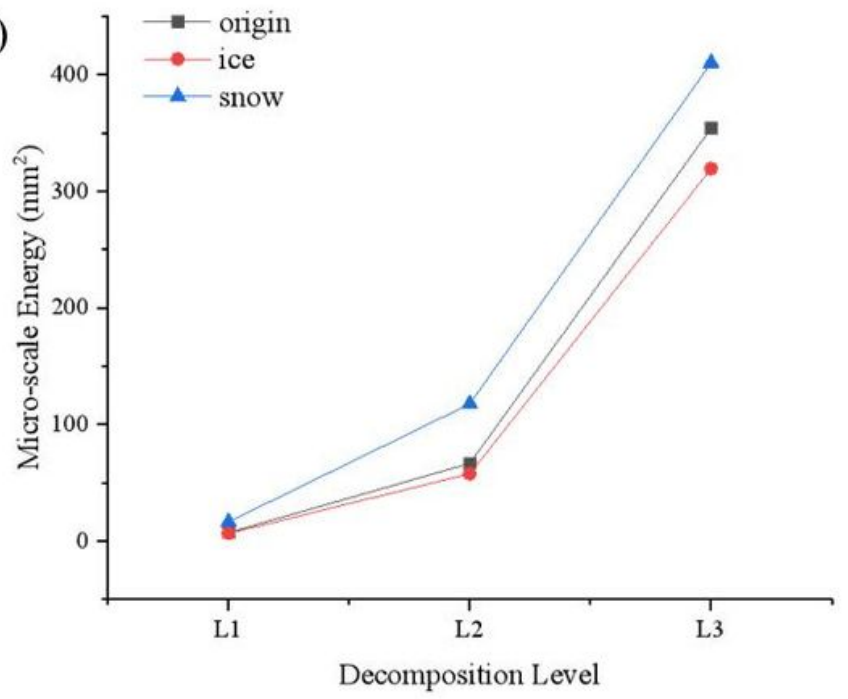

c

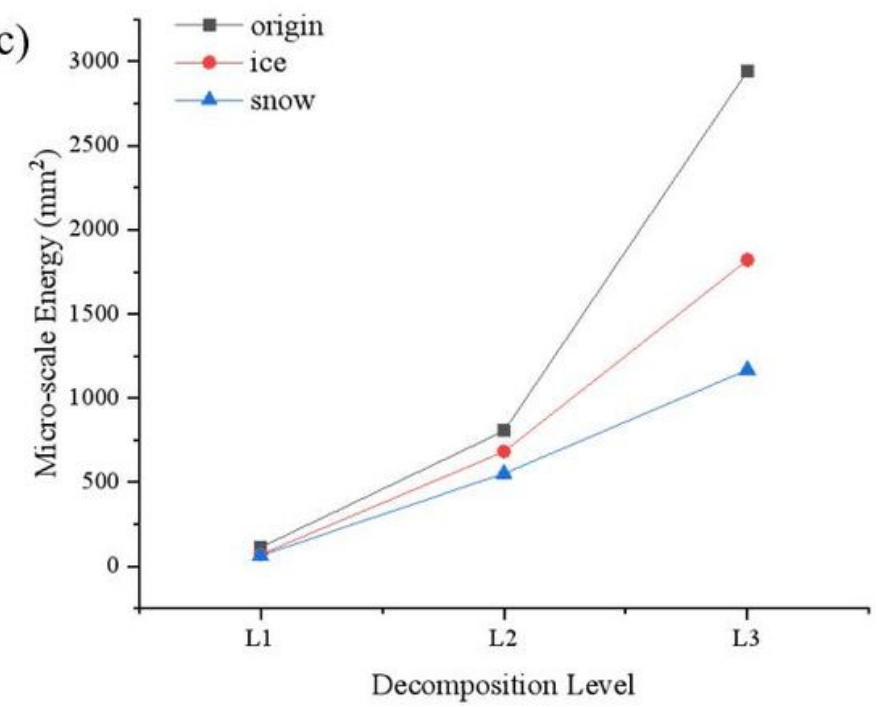

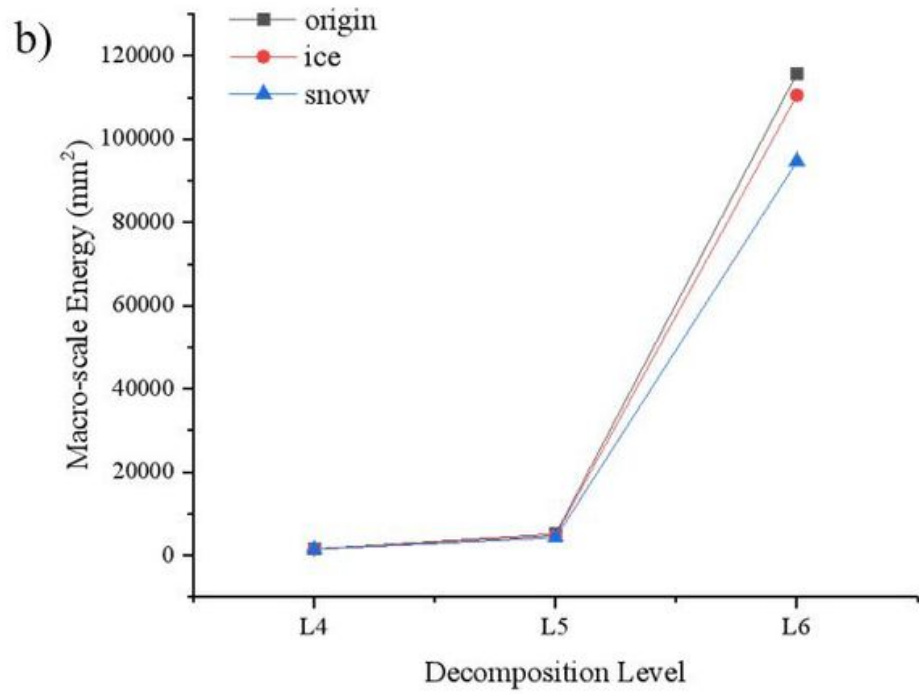

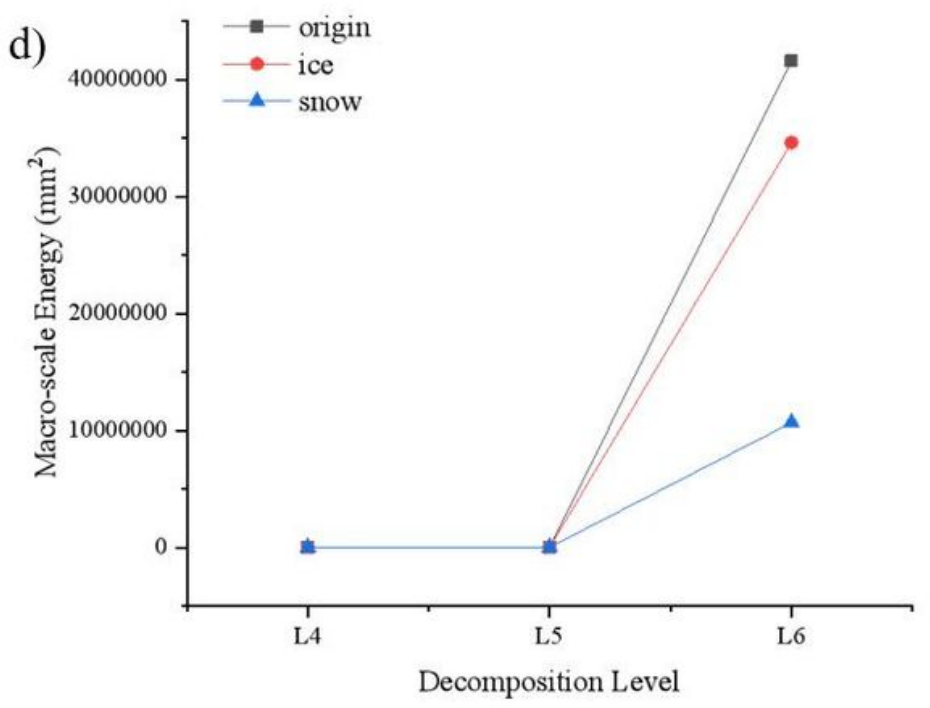

\section{Figure 9}

The Energy Results of AC-16; a) 50\% Cutting Plane in micro-scale, b) $50 \%$ Cutting Plane in macro-scale, c) $100 \%$ Cutting Plane in micro-scale, d) $100 \%$ Cutting Plane in macro-scale. 
a)

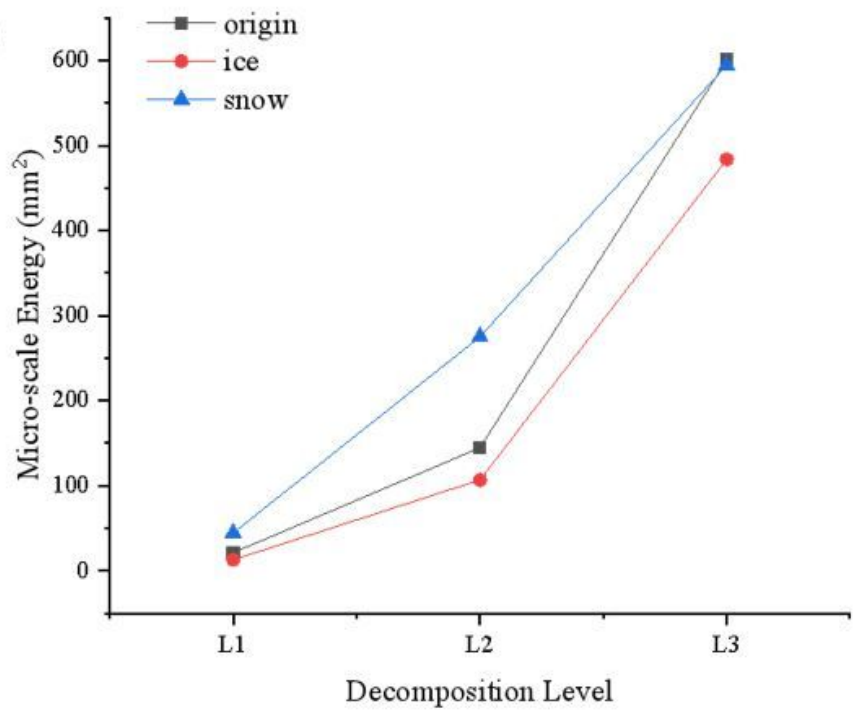

c)

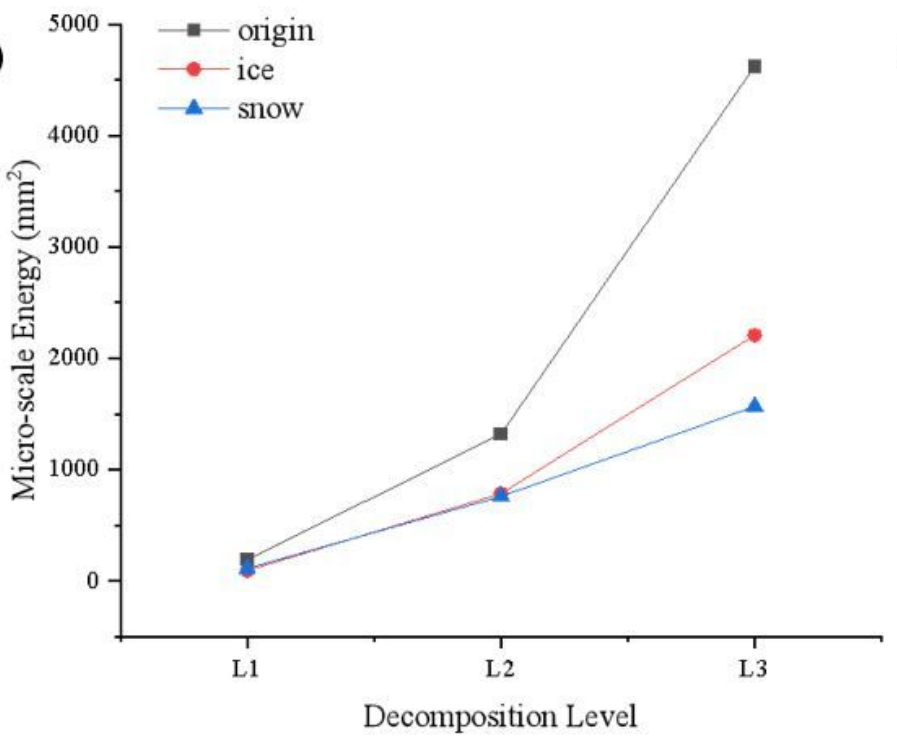

b)

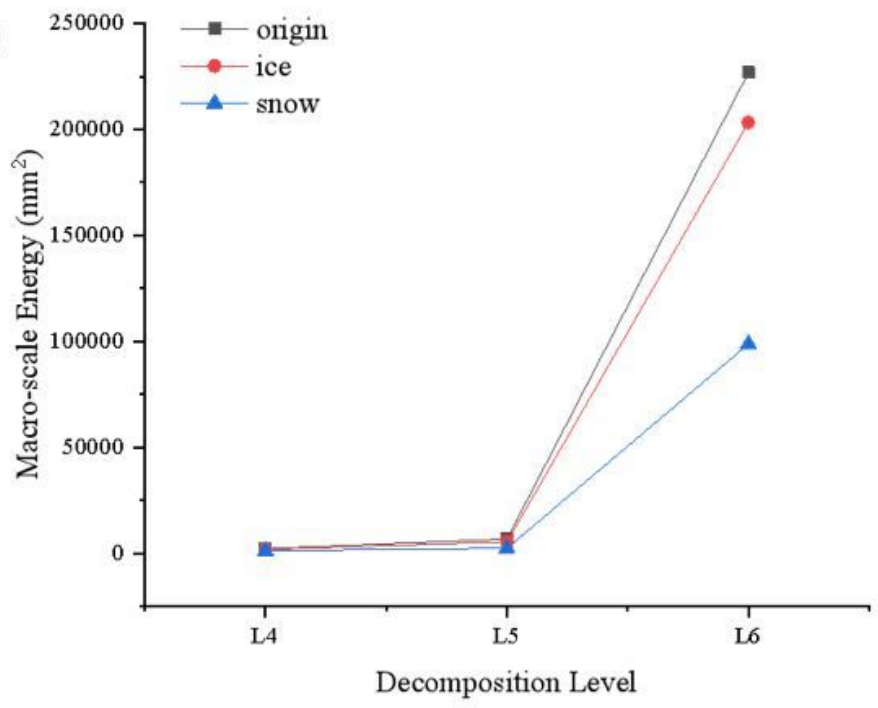

d)

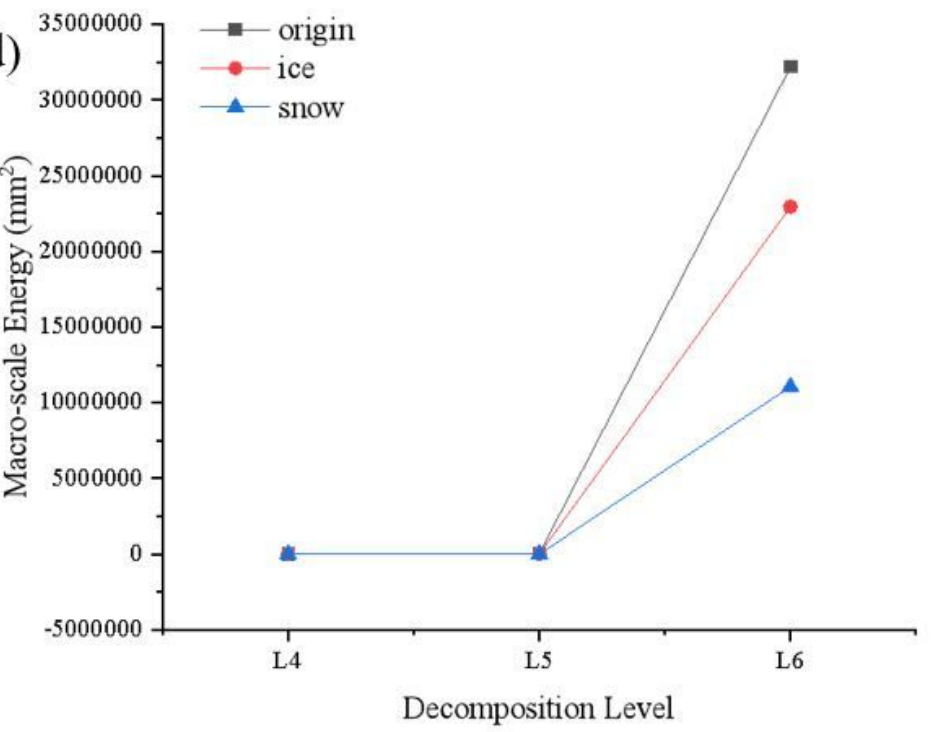

\section{Figure 10}

The Energy Results of SMA-13; a) 50\% Cutting Plane in micro-scale, b) $50 \%$ Cutting Plane in macro-scale, c) $100 \%$ Cutting Plane in micro-scale, d) $100 \%$ Cutting Plane in macro-scale. 

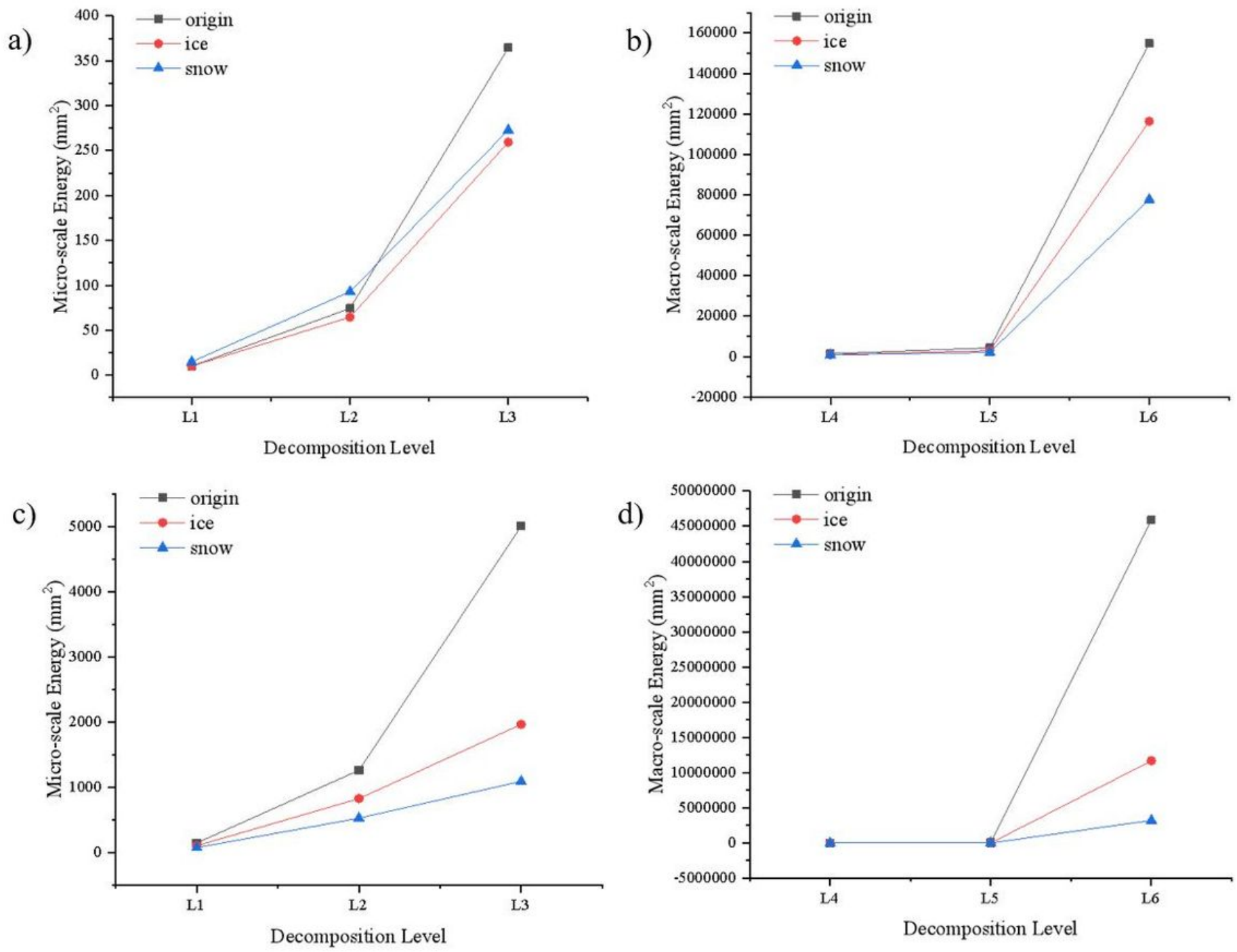

\section{Figure 11}

The Energy Results of SMA-16; a) 50\% Cutting Plane in micro-scale, b) $50 \%$ Cutting Plane in macro-scale, c) $100 \%$ Cutting Plane in micro-scale, d) $100 \%$ Cutting Plane in macro-scale. 

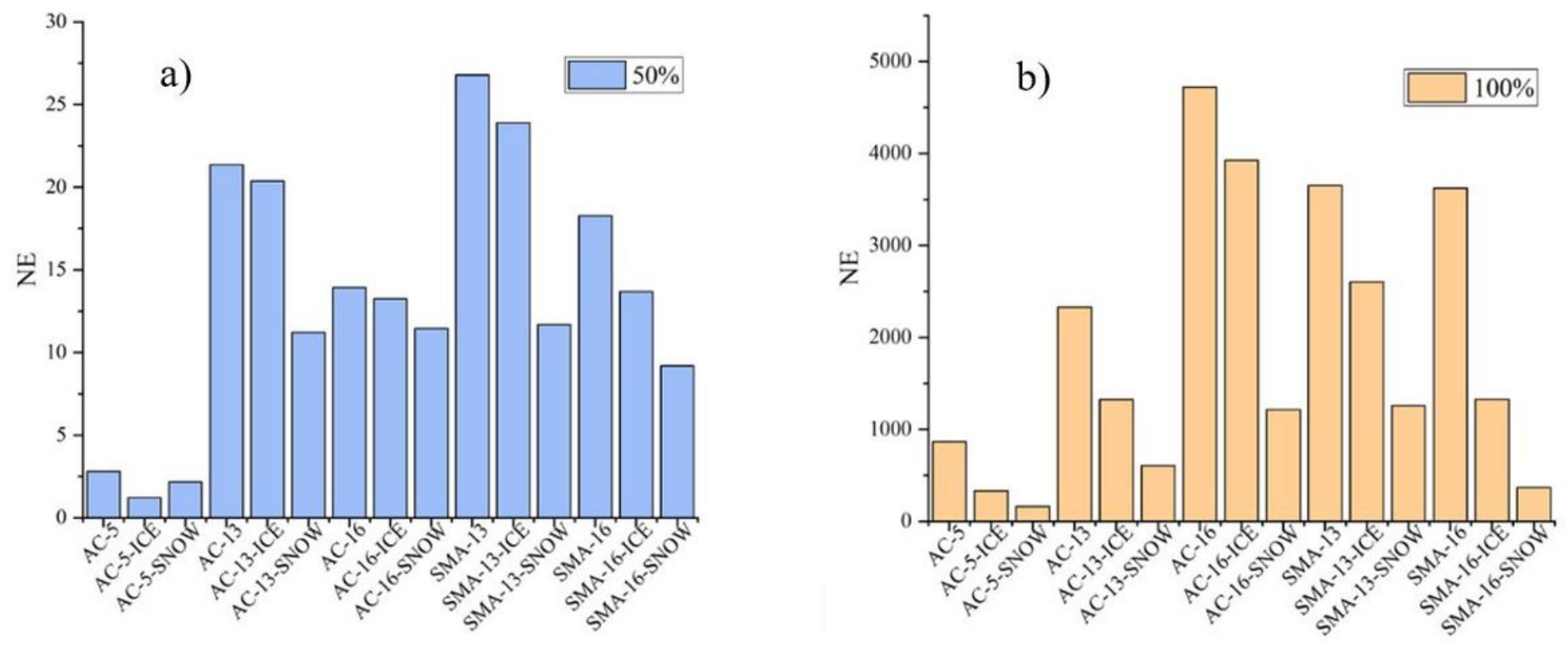

Figure 12

The NE result; a) the NE results on $50 \%$ cutting plane, b) the NE results on $100 \%$ cutting plane. 


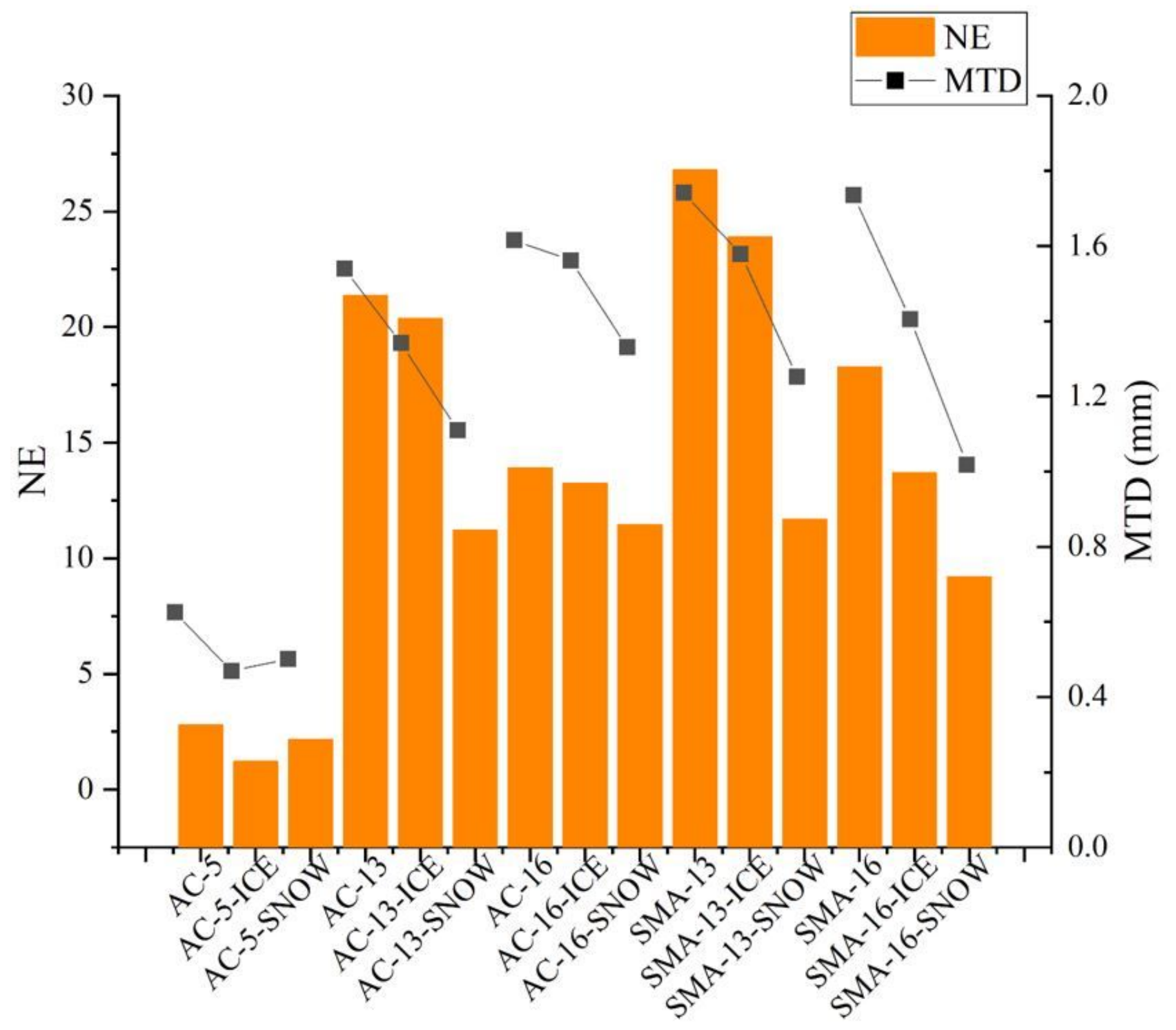

Figure 13

Comparison between MTD and NE for $50 \%$ cutting plane. 